\title{
Band alignment at interfaces of 2-dimensional materials: Internal photoemission analysis
}

\author{
Valery V Afanas'ev, Gilles Delie, Michel Houssa, Ilya Shlyakhov, Andre Stesmans and \\ Vadim Trepalin
}

Laboratory of Semiconductor Physics, Department of Physics and Astronomy, University of Leuven, Belgium

E-mail: valeri.afanasiev@fys.kuleuven.be

Received xxxxxx

Accepted for publication $\mathrm{xxxxxx}$

Published xxxxxx

\begin{abstract}
The article overviews experimental results obtained by applying Internal PhotoEmission (IPE) spectroscopy methods to characterize electron states in single- or few-monolayer twodimensional (2D) materals and at their interfaces. Several conducting (graphene) and semiconducting (transitional metal dichalcogenides $\mathrm{MoS}_{2}, \mathrm{WS}_{2}, \mathrm{MoSe}_{2}$, and $\mathrm{WSe}_{2}$ ) films have been analyzed by IPE, which reveals significant sensitivity of interface band offsets and barriers to the details of the material and interface fabrication indicating violation of the Schottky-Mott rule. This variability is associated with charges and dipoles formed at the interfaces with van der Waals bonding as opposed to the chemically bonded interfaces of three-dimensional semiconductors and metals. Chemical modification of the underlying $\mathrm{SiO}_{2}$ surface is shown to be a significant factor, affecting interface barriers due to violation of the interface electroneutrality.
\end{abstract}

Keywords: two-dimensional material, interface barrier, internal photoemission, band offset

\section{Introduction}

Size scaling of conventional group IV and $\mathrm{A}_{\mathrm{III}} \mathrm{B}_{\mathrm{V}}$ semiconductor devices as well as metal interconnects $(\mathrm{Cu}$, Co) used in integrated circuits rapidly approaches physical and technological limits. As a result, it becomes attractive to explore opportunities offered by alternative conducting and semiconducting materials and heterojunctions. In particular, few-monolayer (ML) thin films of layered two-dimensional (2D) crystals attract significant attention by offering a complete spectrum of possible electronic properties at an atomic thickness scale, i.e., metallic, semiconducting, and insulating behavior. The 2D lattice structure is believed to allow one to control the crystal film thickness on the ML scale with naturally guaranteed lateral homogeneity. At the same time, since in a $2 \mathrm{D}$ crystal all interatomic bonds are saturated within one ML, no broken (“dangling") bond defects are expected to appear at the surfaces or interfaces of ideal 2D crystals with bonding between the layers in a heterojunction occurring via the van der Waals mechanism $[1,2]$.

Furthermore, since in the van der Waals bonded stacks of 2D layers the lattice matching requirements are relaxed, artificial heterojunctions combining dissimilar materials would be permitted with no detrimental mismatch-induced defects. This unique opportunity promises fabrication of device structures suitable for a broad variety of electronic and optoelectronic applications [3 - 7]. For example, fewML $\mathrm{MoS}_{2}$ has already been suggested to enable improvements of metal-insulator-semiconductor (MIS) transistors including channel thickness downscaling, better electrostatic control, and a steep sub-threshold slope [8 15]. The potential of $2 \mathrm{D}$ materials to be combined in 
functional heterostructures has extensively been overviewed in the past [2, 16 - 19] and will not be discussed in the present publication. Here we address the fundamental issue of electron band alignment at interfaces of 2D materials since it essentially determines the transport of electrons across the interface.

It needs to be added here, that realization of technologically feasible devices based on 2D heterojunctions is complicated by the fact that direct growth of the van der Waals bonded stacks is highly problematic: Chemical synthesis of the most common 2D materials, e.g., of graphene or $\mathrm{MoS}_{2}$, requires exposure of the one- or fewML film surface to the reactive ambient at elevated temperatures, often approaching $1000{ }^{\circ} \mathrm{C}$, causing chemical and structural damage [20 - 22]. As a result, the key technology enabling 2D stacking remains solely based on synthesis of a 2D layer on a sufficiently stable substrate $(\mathrm{Cu}$, $\mathrm{Pt}, \mathrm{Ni}, \mathrm{SiC}, \mathrm{SiO}_{2}$, sapphire, etc...), followed by defoliation of the grown film, and next, layer transfer (or, alternatively, ink printing [23]) to the desired target substrate. This transfer approach represents nowadays the only technically feasible way to fabricate van der Waals heterojunctions on a wafer scale and, as a result, is universally used. However, the transfer processing is by far not benign, e.g., it may introduce organic contamination which impairs the electrical properties of the transferred layers [24]. Furthermore, even removal of ambient adsorbates which rapidly form $a \approx 1 \mathrm{~nm}$ thick overlayer [25] may become challenging because of limited thermal stability of, for instance, the chalcogenidebased 2D semiconductor films [20].

Obviously, the atomic and electronic structure of these 'real' interfaces differs significantly from the idealized heterojunctions used in theoretical models in which defectfree surfaces of 2D material are separated from the substrate (or overlayer) only by the van der Waals (vacuum) gap. Though the electrical impact of these non-ideality factors has been well documented over the years [24, 26, 27], more specific information regarding the energy distribution of electron states at the 2D interfaces remains largely unknown since it is still difficult to access it experimentally.

In the present work we overview results of band alignment characterization at the interfaces of one or few ML thin conductors (graphene) and semiconductors (transition metal dichalcogenides, TMDs, such as $\mathrm{MoS}_{2}$ and $\mathrm{WS}_{2}$ ) synthesized by a variety of methods including direct growth and the 2D layer transfer. This comparison enables one to directly estimate the impact of the transfer processing on the electronic properties of 2D semiconductor interfaces, a case which cannot be envisioned in the defoliated flakes being inevitably subjected to the transfer treatment [28].

In order to reliably measure the $2 \mathrm{D}$ semiconductor bandgap edge energies with respect to the bands of other materials we applied spectroscopy of Internal
PhotoEmission (IPE) of electrons from the 2D layer into a thin insulating film underneath [29 - 31]. By studying oneand few-ML thick films of graphene, $\mathrm{MoS}_{2}, \mathrm{WS}_{2}$, and $\mathrm{WSe}_{2}$ synthesized or transferred on top of different insulating materials $\left(\mathrm{SiO}_{2}, \mathrm{Al}_{2} \mathrm{O}_{3}, \mathrm{HfO}_{2}\right)$ as the prototype interfaces, we observed electron IPE from these $2 \mathrm{D}$ photoemitters and determined the corresponding energy barriers. We also addressed the energy barriers at the interfaces of 2Dmaterials with $3 \mathrm{D}$-metals as well as in $\mathrm{MoS}_{2} / \mathrm{WS}_{2}$ and $\mathrm{WS}_{2} / \mathrm{MoS}_{2}$ heterojunctions grown on top of $\mathrm{SiO}_{2}$ and examined environmental effects due to sample storage in room ambient and the impact of the water-based layer defoliation and transfer. The revealed significant sensitivity of the interface energy barriers to the 2D layer processing suggests an important impact of the chemical modification of van der Waals interfaces. This makes the routinely used band edge energies calculated in idealized heterojunctions or their positions measured under vacuum conditions irrelevant to the stacked device structures fabricated using wafer-scale 2D layer transfer. On the other hand, the IPE-based interface barrier metrology is shown to deliver not only information regarding the interface barrier heights but also can be used to determine onsets of optical electron excitations in 2D semiconductors [32], thus enabling determination of both the conduction band (CB) and the valence band (VB) offsets at their interfaces.

\section{Impact of the band alignment on electronic properties of 2D- heterojunctions}

Before describing the experimental methodology of IPE and overviewing available experimental results regarding 2D-materials interface band offsets, let us discuss the electrical properties of heterostructures which are directly influenced by the energy barriers mobile charge carriers encounter at interfaces. Since the control of electron transport is based on two functionalities - charge injection and charge insulation, - two different requirements for the interface barrier height become self-evident: Efficient injection of charges would require a low and narrow barrier to ensure a high electron tunneling rate in a field-emission mode and the corresponding Ohmic behavior [33]. By contrast, in the case of an insulating interface the height of the barrier should be maximized to ensure a low electron tunneling rate even in the case of few-nm thick barriers typical for nano-scale electron devices [34].

\subsection{Contact resistance and Schottky barriers}

Electron injecting contacts are of primary importance for field-effect transistor (FET) devices since the contact resistance $R_{c}$ is in series connection with the semiconductor channel and represents the major limiting factor in achieving a high ON-state current density. In particular, for 2D TMD 
semiconductors the contact resistances appear to be high which was attributed to Fermi level pinning due to chemical interaction between the sputtered 3D-metal and the 2Dsemiconductor surface layer [35]. Reduction of the contact resistance is often observed with increasing transversal electric field in the transmission line method (TLM) [36, 37]. This behavior suggests formation of a depletion layer in the near-contact semiconductor region. To avoid chemical interaction between the contact metal and the semiconductor, two approaches are currently considered. First, a "gentle" evaporation of a low-reactivity metal, e.g., Indium in combination with an Au protecting cap, helps to prevent interatomic bond rupture at the semiconductor surface [38, 39]. However, despite delivering low resistivity contacts to $\mathrm{MoS}_{2}$, these In/Au stacks lack sufficient thermal stability. Alternatively, insertion of a graphene layer between the contact metal and the $2 \mathrm{D}$ semiconductor has been shown to reduce the Schottky barrier height (cf. Fig. 4 in Ref. 2). This effect has been ascribed to the prevention of metal atoms reacting with the semicondutor surface [40] resulting in "an ultra clean and flat interface formation at the graphene-2D semiconductor junction with no chemical bond breaking, thus suppressing the Fermi-level pinning" [17]. This explanation is still questionable since experiments with a graphene layer inserted between the contact metal and the 3D semiconductor crystal (silicon) indicate that the Fermi level remains pinned despite the absence of intermixing between $\mathrm{Si}$ and the metal [41]. Other contact types, e.g., the rectifying (Schottky) barrier diodes with a rectification ratio as high as $10^{7}$ at $300 \mathrm{~K}$ have recently been reported [42].

In all cases determination of the barrier height is critically important to estimate the potential of using a particular metallization scheme to achieve low $\mathrm{R}_{\mathrm{c}}$ [38] or, else, high non-linearity required for selector diodes [43, 44]. In particular, the lateral variability of the barrier height becomes increasingly noticeable in layered chalcogenide semiconductors [45 - 47]. Since measurements of the depletion layer capacitance which provide the most accurate way of the Schottky barrier height determination [48] become impossible in one- of few-ML thin films, one should opt for current measurements. In this case IPE represents the optimal choice since contributions of sample regions with different barrier heights can be spectrally resolved $[49,50]$. At the same time, thanks to using low-energy photons, IPE allows one to avoid charging artifacts inherently caused by ionizing radiation when using $\mathrm{X}$-rays to excite photoemission into vacuum [51].

\subsection{Insulating interfaces and resistive switching}

The formation of an interface barrier of sufficient height at interfaces of 2D materials is critically important for the realization of field-effect devices in which the charge carrier density in the transport channel is controlled by an externally applied electric field, see, e.g. the planar $\mathrm{MoS}_{2}$ FET structure in Ref. 8. Reliable insulation at room temperature requires that the barriers for electrons or holes in the 2D material exceed $2 \mathrm{eV}$. Since the electron injection rate over such barrier in thermal equilibrium is negligible, one needs to provide additional energy to electrons externally, e.g., by optical excitation, to make their transitions over the barrier observable. Again, IPE offers the possibility to observe these transitions directly without introducing additional charges to the insulating layer or to the interfaces of 2D materials [30].

Another application in which the insulating function of 2D interfaces becomes important concerns the resistive switching memory cells $[52,53]$. Use of the $2 \mathrm{D}$ materials as a dielectric switching medium, e.g., h-BN, or as a highly stable electrode material (such as graphene) precluding interdiffusion [54], also requires engineering of interface barrier heights thus mandating quantification of the latter.

\subsection{Tunnel transport}

Another physical phenomenon in which electron transport is controlled by the interface barrier height is electron tunneling. Thanks to the van der Waals bonding, few-ML thin 2D layered materials with a sizable bandgap and appropriate band alignment can be combined in heterojunctions without lattice matching constraints and thus potentially excluding the layer thickness variability [5]. Based on the electron tunneling, a broad spectrum of electron devices has been proposed, ranging from Esaki diodes delivering a negative differential resistance $[5,55]$ to tunnel FETs promising a steep subthreshold slope $[13,56]$ which is critical for low-voltage operation. Since the tunnelling barrier height and width affect the electron tunneling probablity exponentially, understanding the mechanism of barrier formation and trends in band alignment at the interfaces of 2D materials becomes crucial for the development of these devices.

\subsection{Built-in potentials and device electrostatics}

Another important factor associated with the interface band alignment is the built-in voltage arising from the contact potential difference between stacked layers of dissimilar materials. This built-in potential influences the electronic properties of heterojunctions in a variety of ways. First, the built-in potential adds to the externally applied bias and directly affects the threshold voltage of a FET. Obviously, to enable low voltage circuit operation one needs to tightly control the effectve work functions (EWFs) of materials in contact. The latter are not necessarily the same as the work function (WF) of a clean metal or semiconductor surface and may contain additional contributions due to the possible violation of the interface 
electroneutrality caused by charge transfer between layers [50]. Furthermore, in the case of van der Waals bonded heterojunctions, both sides of the interface are accessible for adsorbates and contaminants [27] which may have a doping effect. The latter means that there will be a charged layer at the interface between the two films which will electrostatically shift their bands. Variations in the interface charge density may be induced by different processing steps, including, for example, transfer of a 2D layer on top of the target substrate [28] or by changing the material synthesis chemistry [57] making the interface barriers as well as the contact potential differences poorly reproducible and sensitive to the environment.

Second, the electric field associated with the contact potential difference remains present even when the external voltage source is disconnected. Taking into account the EWF differences which can reach $\approx 1 \mathrm{eV}$ and combined with the thickness of one-ML 2D material in the range less than 1 $\mathrm{nm}$, the strength of the built-in electric field may approach up to $10 \mathrm{MV} / \mathrm{cm}$. It is well possible that this field will cause atomic motion (kind of electromigration) of ionized species, as mentioned above, leading to the long-term instabilities. For example, variation of the electrode metal workfunction in resistive switching cells has a pronounced effect on the retention properties [58]. On the other hand, the presence of a significant built-in potential is essential for photovoltaic devices [17].

\section{Physics of $2 \mathrm{D}$ interface barriers}

The current understanding of physical factors determining the interface band alignment in solid heterojunctions has been extensively overviewed in the literature, see, e.g., reviews of R. T. Tung [59, 60]. Starting from the idealized interface model "without chemistry" [60] one can include additional factors in a stepwise manner and estimate their relative significance. In this way the band alignment and interface barriers of $2 \mathrm{D}$ materials are expected to be determined by similar physics as in the case of interfaces of three-dimensional solids.

\subsection{Schottky-Mott model and band offset transitivity}

The zero-order approach to the estimation of the metalsemiconductor barrier height (the Schottky barrier) or band offsets at semiconductor interfaces consists in omitting interface-specific interactions and charge imbalances. This picture, often referred to as the Schottky-Mott rule [59, 60], assumes that the electron band alignment is determined by the energies of these bands inside isolated crystals. Therefore, the interface barriers can be obtained by aligning the band edges with respect to the common vacuum level
$\mathrm{E}_{\mathrm{vacuum}}$, for example, the conduction band offset $\Delta \mathrm{E}_{\mathrm{C}}$ can be calculated as a difference between electron affinities $(\chi)$ of two solids (the Anderson rule for semiconductor heterojunctions [61]) as shown in figure 1 .

As a consequence of omitting interface effects the "transitivity" principle of semiconductor band offsets has been formulated [59]. According to this rule, for any three semiconductors, $\mathrm{A}, \mathrm{B}$, and $\mathrm{C}$, the sum of the conduction or valence band offsets $\left(\Delta \mathrm{E}_{\mathrm{V}}\right)$ at interfaces $\mathrm{AB}, \mathrm{BC}$, and $\mathrm{CA}$ should be equal to zero as schematically illustrated in figure 1. Quite surprisingly, experiments show that this idealized description is relevant for a rather wide spectrum of interfaces between crystalline semiconductors and between semiconductors and oxide insulators [30]. No impact of a dissimilar interlayer on the interface barrier height has been found in several semiconductor heterojunctions [62]. Even in the case of polar semiconductor (GaAs) surfaces, the interface barrier with an oxide insulator $\left(\mathrm{Al}_{2} \mathrm{O}_{3}\right)$ appears to be insensitive to the crystal surface orientation [63]. This observation suggests a compensation of the dipole related to the array of polar chemical bonds established at the surface of the semiconductor by the oppositely oriented dipole in the next atomic layer. If now turning to the interfaces involved with van der Waals bonding which can be seen, in an ideal case, as two solid surfaces separated by a vacuum gap, the barrier behavior is expected to differ significantly from the above discussed interfaces realized with chemical bonding.

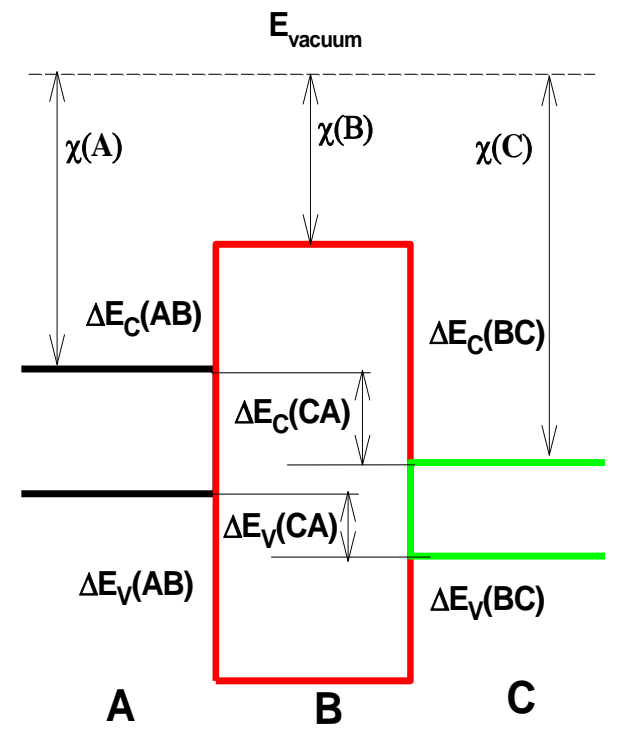


Figure 1. Schematic illustration of the band offset transitivity principle and its relationship to the Schottky-Mott (electron affinity) rule.

\subsection{Interface dipoles, polarization layers, and dopants}

Deviations from the Schottky-Mott rule are well documented and usually ascribed to the violation of electroneutrality at the interface due to incorporation of charges or additional dipoles (see [59,60] and references therein). A similar list of non-idealities has also been proposed for interfaces of 2D materials such as contacts with 3D metals [64]. First of all, there are charged interface states associated with processing-induced damage of 2D materials (e.g., metallization sputtering [64] or plasma exposure [65]) and chemical reactions at the interface [64, 66] which cause Fermi level pinning. Next, one may also expect intrinsic contributions due to "tailing" of the electron density into the van der Waals gap (Shockley-Tamm states) [64]. Interface charges will also be sensitive to extrinsic factors such as adsorbates and contaminants remaining at the interface after exfoliation of the flake or the layer transfer. In particular, adsorption of polar molecules $\left(\mathrm{H}_{2} \mathrm{O}\right)$ may affect the effective work function and the Schottky barrier height [67]. In a similar way, an additional change of the electrostatic potential can be introduced by remote doping used to increase the charge carrier density in graphene and 2D semiconductor layers [68 - 70].

To this it should be added that the surfaces of insulating materials $\left(\mathrm{SiO}_{2}\right.$, high-permittivity oxides, h-BN, mica...) used as substrates to fabricate electron devices based on 2D materials are also prone to interaction with adsorbates which may lead to formation of near-interface charges [71, 72]. This charged layer, often referrred to as "polarization layer" [73], also contributes to the violation of the interface electroneutrality leading to the sensitivity of the effective work function and the electron affinity of 2D films to the type of substrate used. All in one, one may expect the interface band alignment at the van der Waals bonded interfaces of $2 \mathrm{D}$ materials to be highly sensitive to the processing conditions and to the environment.

\section{Band alignment characterization by using internal photoemission}

As to 3D solids, the basic physics of IPE and its application for the determination of interface barriers and band offsets have been overviewed previously [29 - 31]. Here we shall focus specifically on the features that make IPE spectroscopy the method of choice to characterize interfaces of $2 \mathrm{D}$ materials. In applying IPE, to be noted is that the few-ML thickness of a $2 \mathrm{D}$ photoemitter may also affect the physical description of IPE by modifying electrostatic interactions in the barrier region as well as requiring transition to the $2 \mathrm{D}$ model for the density of electron states (DOS) at the emitter surface. Finally, a novel approach to photoconductivity (PC) measurements will be discussed which allows one to circumvent the sensitivity problem caused by the high charge carrier recombination rate in few-ML thin films.

\subsection{Interface-specific effects in IPE}

As opposed to the photoemission of electrons into vacuum from the surface of an emitter, in the case of IPE electrons cross the interface with another solid having different atomic structure and, as a consequence, a different distribution of the electrostatic potential. As a result, in addition to the "bulk" electronic structure contribution (in the Schottky-Mott sense $[59,61])$ the vacuum WF will be affected by the surface dipole barrier component [74] while the EWF shall reflect chemical bonding at the particular interface and the charges/dipoles present there. In other words, the vacuum WF represents the property of a certain atomic surface while the EWF may also depend on the properties of the second component of the interface. Therefore, one cannot expect that these quantities will be equal but the EWF (or electron affinity in the case of a semiconductor) of the same material will be sensitive to the type of interface and to the process of its fabrication. For this reason, interface barriers inferred from IPE results provide by far a more reliable estimate of the EWF than the vacuum WF values. Differences can easily exceed $0.5 \mathrm{eV}$ [50].

Next, by avoiding the use of ionizing radiation (high energy UV, X-rays) to excite photoemission, IPE into insulating substrates is not suffering from the long known "differential" charging artifacts [75 - 77]. Furthermore, charges trapped in the insulating layer upon X-ray exposure are known to dope $2 \mathrm{D}$ materials $[69,78]$, i.e., the measurement procedure used in the $\mathrm{X}$-ray photoelectron spectroscopy may change the mobile carrier concentration and the Fermi level position which directly affects the EWF.

Finally, thanks to the possibility of applying an external electric field during the IPE experiment, one can characterize the kind of electrostatic perturbation responsible for the deviation from the ideal Schottky-Mott behavior: In the case of an interface dipole the field-induced barrier lowering still follows the Schottky law [79, 80] while introduction of charges is expected to cause deviations from the image-force barrier behavior [73, 81].

4.2 Photoemission from $2 \mathrm{D}$ solids 
The transition from a 3D solid to a 2D few-ML thin photoemitter can be expected to change the conventional description of the IPE spectral dependences [82] by facts such as the transition to the 2D DOS of the initial electron states and the cancelation of the condition of transversal crystal momentum conservation which, in the absence of translational symmetry in this direction, cannot be considered to remain a "good" quantum number. Furthermore, in the case of IPE from a one or few ML thin film the average photoelectron escape depth will be detemined not by scattering but by the physical thickness of the photoemitter which might affect the shape of the potential barrier at the interface [see Eq. (2.9) in Ref. 81].

Nevertheless, there is no reason to believe that Powell's description of the IPE process [82] needs to be fundamentally modified. For example, the energy distribution of electrons emitted from a one ML 2D semiconductor such as $\mathrm{MoS}_{2}$ [83] and $\mathrm{WS}_{2}$ [84] into vacuum indicates that the DOS in the semiconductor VB increases linearly with energy within a $\approx 1 \mathrm{eV}$ interval below the VB top similarly to the case of photoemission from a bulk Si crystal [85]. According to the Powell model [82], such DOS would correspond to the dependence, above the spectral threshold $\Phi_{\mathrm{e}}$, of the electron IPE quantum yield $\mathrm{Y}$ on photon energy $\mathrm{h} v$ as given by the power function $\mathrm{Y}(\mathrm{h} v) \sim\left(\mathrm{h} v-\Phi_{\mathrm{e}}\right)^{3}$ in agreement with experimental observations [57, 86, 87]. Apparently then, the analysis of IPE spectra for 2D photoemitters can be done in a similar way as carried out for the conventional 3D material interfaces.

In turn, the field-dependent image force barrier model [82] in many cases describes the IPE threshold behavior reasonably well even for $2 \mathrm{D}$ photoemitters [86 - 88]. Therefore, it is still logical to use this model to account for the image-force barrier lowering and determine the true barrier height by extrapolating the field-dependent IPE threshold to zero field strength. Deviations from the ideal Schottky behavior can be observed in IPE both from 3D and $2 \mathrm{D}$ materials and, as mentioned above, indicate the the presence of additional electric fields in the barrier region $[50,73,81]$.

\subsection{Lateral non-uniformity of interface barrier}

One of the important complications encountered in the analysis of interfaces of 2D materials consists in the presence of the possible lateral non-uniformity of their electron structure. This non-uniformity may be caused by non-uniformity of the 2D film itself, e.g., co-presence of regions with different thicknesses (different number of MLs), defects (pores, transfer-induced cracks), patches of contaminants [24], non-uniformity of the underlying substrate, etc. Evaluation of this non-uniformity becomes important when considering the corresponding variability of the built-in potential which induces an electric field directly affecting the electron transport in the vicinity of the interface $[89,90]$. A the traditional way to estimate these non-uniformities is based on measurements of the capacitance of the semiconductor deplection layer [91, 92]. Besides being limited to the interfaces of low-doped semiconductors, this approach obviously fails if the semicondcutor thickness approaches the ML range. On the other hand one can attempt to simulate the electron injection current across a non-uniform interface but this method fails to provide a unique answer regarding the distribution in barrier height [93].

While in the case of surfaces the problem is solved using scanning probe microscopy (see, e.g., [94]), IPE probably provides the most straightforward way to estimate interface barrier non-uniformity. This can be done either by direct observation of different spectral thresholds [49] or using contact potential difference with laterally uniform, e.g., a single-crystal reference electrode in a capacitor structure [50]. This is of particular importance for few-ML thin 2D electrodes which the continuity of the film cannot be $a$ priory guaranteed: If evaporating on top of such film a semitransparent layer of a contact metal with a low EWF, one can observe IPE from both materials and estimate areal fraction of pores in the 2D film from the intensity of wellresolved IPE from the metal.

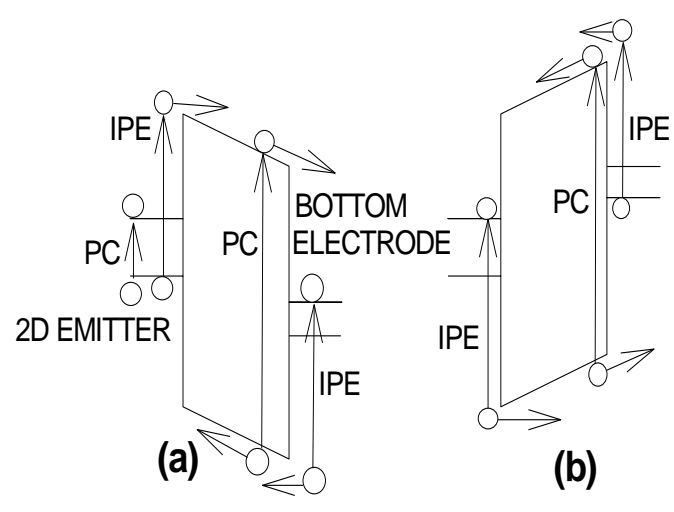

Figure 2. Band diagrams showing schematic of IPE- and PC-type optical electron transitions in a2D photo emitter/insulator/bottom electrode (e.g., $\mathrm{Si}$ ) structure potentially observable under negative 
(a) and positive (b) bias voltage applied to the 2D semiconductor electrode.

\subsection{Photoconductivity of $2 D$ materials}

As one can see from the band diagram shown in figure 2, illustrating the possible electron transitions in a $2 \mathrm{D}$ photoemitter/insulator/Si bottom electrode structure, by using spectrally-resolved PC measurements one can determine the most important electron structure parameter of a semiconductor - the bandgap width $\mathrm{E}_{\mathrm{g}}$. Combining this value with the observed threshold, $\Phi_{\mathrm{e}}$, of electron IPE from the semiconductor 2D photoemitter VB allows one to determine, for example, the $\mathrm{CB}$ offset at the interface with insulator or the Schottky barrier height for minority charge carriers. In the case of sufficiently thick semiconductor layers, the PC can be directly measured as a photocurrent across the interface (in diode structures) or through an illumination-induced variation of the depletion layer width (at interfaces with insulators). Yet, the typically small thickness of 2D electrodes (in the few ML range) limits light absorption and thus hampers sensitivity of these measurements.

When a semiconductor layer is grown on top of or transferred to an insulating substrate, the lateral drift of photogenerated charge carriers can be used to observe PC in the co-planar geometry. The value of the photocurrent in this case is strongly influenced by the surface recombination rate and rapidly decreases with increasing photoconductor length. For CVD- grown 2D semiconductors such as $\mathrm{MoS}_{2}$ and $\mathrm{WS}_{2}$ the spectrally-resolved $\mathrm{PC}$ can be detected in samples with length less approximately $1 \mu \mathrm{m}$, thus necessitating lithographic processing. The latter cannot be considered to be benign if the thickness of the semiconductor film is scaled down to a single ML, being troubled by possible influences of contamination, strain, and delamination of the weakly (van der Waals) bonded layer.

Yet, photogenerated electron-hole pairs in non-patterned 2D semiconductor films can be detected in a simple capacitor set up by measuring the displacement current associated with trapping of charge carriers by defects outside the semiconductor layer, e.g., by traps in the underlying insulator or in the interlayer between the 2D film and the substrate. The applicability of this method, which can be considered as "the IPE to traps", to single-ML $\mathrm{MoS}_{2}$ and $\mathrm{WS}_{2}$ has recently been demonstrated [32]. An additional advantage of this measurement scheme consists in the possibiliy to observe charge carrier generation in a singleML semiconductor covered by relatively thick (10-20 nm) semitransparent metal electrode, allowing one to reveal the impact of metallization on the electron states of the semiconductor. Furthermore, using this metallization scheme, it becomes possible to detect optical generation of charge carriers in discontinuous semiconductor layers -an achievement which cannot be realized in another way. Finally, the samples for this "transversal" PC detection are identical to those used in IPE, i.e., the PC and IPE transitions are detected in one photocurrent spectral curve recorded over an extended photon energy range.

\subsection{Experimental realization}

In the IPE studies we focussed on several wafer-scale synthetic 2D materials (one- and few- ML graphene, $\mathrm{MoS}_{2}$ and $\mathrm{WS}_{2}$ ) of sufficient stability in air, which makes them the most viable candidates for use in practical electron devices. Furthermore, comparison between films synthesized on top of $\mathrm{Si} / \mathrm{SiO}_{2}$ structures with the same semiconductors transferred on top of identical $\mathrm{Si} / \mathrm{SiO}_{2}$ entities allows one to reveal the impact of the layer transfer processing on the electronic properties of the interface.

Monolayer or few-layer graphene has been synthesized by the chemical vapor deposition (CVD) method on a $\mathrm{Pt}(111)$ template at $1070{ }^{\circ} \mathrm{C}$ using a gas mixture of $\mathrm{CH}_{4} / \mathrm{H}_{2}=$ $8 / 850$ followed by transfer onto a target substrate - p-type $\mathrm{Si}(100)$ covered with $50 \mathrm{~nm}$ thermal $\mathrm{SiO}_{2}$. The template was initially grown on a c-plane of a sapphire crystal to achieve a high-quality Pt foil with low grain boundary density. To release graphene from the growth template onto a target substrate water intercalation between graphene and Pt layers was used [95]. This was followed, successively, by the layer transfer process involving polymethyl metacrilate (PMMA) spin coating, peeling off graphene with PMMA from Pt, transfer of it onto the $\mathrm{Si} / \mathrm{SiO}_{2}$ target substrate and PMMA removal in acetone and isopropyl alcohol. Finally, to eliminate the excess water, the sample was annealed at $400{ }^{\circ} \mathrm{C}$ for $4 \mathrm{~h}$ in vacuum or in forming gas $\left(\mathrm{N}_{2}+10 \% \mathrm{H}_{2}\right)$.

Several technologies of synthesis and transfer of oneand few-ML thin films of transition-metal di-chalcogenides (TMDs), $\mathrm{MoS}_{2}$ and $\mathrm{WS}_{2}$, were compared. First, we addressed films synthesized using the metal sulfurization technique $[28,88,96,97]$. The synthesis starts from thermal evaporation of few- $\AA$ thin Mo or $\mathrm{W}$ films on $200 \mathrm{~mm}$ $\mathrm{SiO}_{2}(50$ or $90 \mathrm{~nm}) / \mathrm{Si}$ or $150 \mathrm{~mm}$ c-plane sapphire substrate wafers under high vacuum $\left(10^{-6} \mathrm{mbar}\right)$ at a deposition rate of $0.01 \mathrm{~nm} / \mathrm{s}$. Then the samples were annealed in pure $\mathrm{H}_{2} \mathrm{~S}$ atmosphere at a pressure of $10 \mathrm{mbar}$ at $800^{\circ} \mathrm{C}\left(\mathrm{Si} / \mathrm{SiO}_{2}\right.$ substrates) or $1000^{\circ} \mathrm{C}$ (sapphire substrates). Besides allowing one to compare band alignments at interfaces with $\mathrm{SiO}_{2}$ for two different metal cations (Mo and W) this approach can also be used to synthesize $\mathrm{WS}_{2} / \mathrm{MoS}_{2}$ and $\mathrm{MoS}_{2} / \mathrm{WS}_{2}$ heterojunctions on top of the insulating layer [96].

Alternatively, one- and few-ML $\mathrm{MoS}_{2}$ and $\mathrm{WS}_{2}$ films were grown on top of the $\mathrm{SiO}_{2}$ at $800{ }^{\circ} \mathrm{C}$ from $\mathrm{Mo}(\mathrm{CO})_{6}$ and $\mathrm{W}(\mathrm{CO})_{6}$ precursors, respectively, following the CVD 
process described elsewhere [98]. As an alternative to this CVD chemistry some layers were synthesized by first depositing $\mathrm{MoO}_{3}$ on top of $\mathrm{SiO}_{2}$ using the $\mathrm{C}_{12} \mathrm{H}_{30} \mathrm{~N}_{4} \mathrm{Mo}$ precursor and oxygen plasma [99] followed by thermal sulfurization in $\mathrm{H}_{2} \mathrm{~S}$ at $900{ }^{\circ} \mathrm{C}$. These films were compared to $\mathrm{MoS}_{2}$ layers grown in $\mathrm{C}$-free and $\mathrm{H}$-free conditions using slow magnetron sputtering of a Mo target in an elemental $\mathrm{S}$ vapour [100].

Some of the grown $\mathrm{MoS}_{2}$ layers were subsequently transferred onto 50- or 90 -nm thick $\mathrm{SiO}_{2}$ thermally grown on (100)Si substrates. The water intercalation-based tape assisted transfer method was used starting from the delamination of the $\mathrm{MoS}_{2}$ film from the growth substrate ( $\mathrm{Si} / \mathrm{SiO}_{2}$ or sapphire). This was followed by bonding of the $\mathrm{MoS}_{2}$ layer on the target $\mathrm{Si} / \mathrm{SiO}_{2}$ wafer surface. Initially, a PMMA layer is spin-coated over the entire $\mathrm{MoS}_{2}$ surface. A rigid thermal tape (REVALPHA $3195 \mathrm{~V}$ ) is placed over the desired area of several $\mathrm{cm}^{2}$ of the PMMA/MoS $/$ substrate stack and then immersed in deionised water at $80{ }^{\circ} \mathrm{C}$. After ultrasonic treatment, the tape/PMMA/MoS 2 stack is slowly peeled off and separated from the growth substrate. Next, the stack is removed from water, blow-dried with $\mathrm{N}_{2}$ and then placed on the target surface. The sample is put on a hot plate at temperature of 125 or $140{ }^{\circ} \mathrm{C}$ to allow the thermal tape to be peeled off from the protective PMMA layer. To remove PMMA the samples were wet-cleaned using isopropyl alcohol and hot acetone. The post-transfer annealing treatments were carried out for $15 \mathrm{~min}$ at $370^{\circ} \mathrm{C}$ under a $\mathrm{H}_{2} \mathrm{~S}$ pressure of 10 mbar.

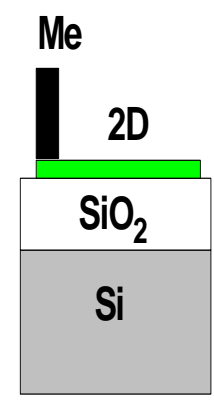

(a)

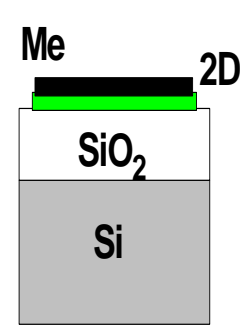

(b)

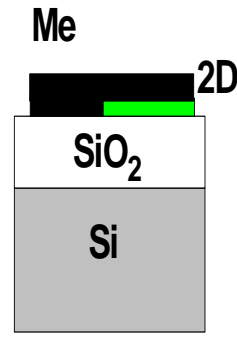

(c)
Figure 3. Metallization schemes used to observe IPE from the uncapped surface of a 2D material (a), from the metallized 2D layer (b) and to simultaneously observe IPE from the 2D emitter and from the metal overlayer (c).

To ensure reliable electrical contacts to the few-ML thick top 2D electrode several contact schemes were used as illustrated in figure 3. First, optically non-transparent (100$\mathrm{nm}$ thick) $\mathrm{Au}$ or $\mathrm{Al}$ pads $\left(0.01 \mathrm{~mm}^{2}\right.$ area) were thermoresistively evaporated on top of the film through a shadow mask leaving the graphene or the TMD surface intact. Alternatively, semitransparent (13-nm thick) metal electrodes of $0.5 \mathrm{~mm}^{2}$ area were evaporated to study contacts between the $2 \mathrm{D}$ material and $3 \mathrm{D}$ metal using overlayer or the edge contact geometry. The steady-state value of the photocurrent was measured between the $\mathrm{Si}$ substrate and the overlayer by applying a negative bias to the latter, thus enabling electron photoinjection when illuminating the sample by monochromatic light with known photon energy hv [29, 30]. The quantum yield $\mathrm{Y}$ is calculated by normalizing the photocurrent to the incident photon flux. Then the spectral dependences of the yield are analyzed to determine the spectral thresholds of IPE which directly correspond to the interface barrier heights.

\section{Internal photoemission at interfaces of graphene}

The weak optical absorption and small thickness of the photo-excited layer make the observation of IPE from single-ML 2D materials challenging since the IPE-related photocurrents are low. This, for instance, precludes meaningful spectrally-resolved IPE experiments on flakes transferred on top of insulating layers mandating use of large-area synthetic graphene. Though the CVD-grown graphene sheets are already available for a while, the problems met in observing electron IPE from this material provide a good illustration of the difficulties mentioned above.

\subsection{Intrinsic band alignment and effective work function}

To the best of our knowledge, the first attempts to measure the band alignment between graphene and $\mathrm{SiO}_{2}$ using IPE spectroscopy have been made by Yan et al. $[101,102]$, but due to the optical transparency and the very limited photoexcited carrier density in a single atomic layer of graphene, no photoemission from graphene could be observed given the detection limit of their measurements as indicated in a later publication of this group [103]. Instead of observing electron IPE from graphene, IPE of holes from the substrate has been detected. This observation had led the authors to the proposal of using graphene as a highly transparent electrode to detect IPE from the bottom electrode in capacitor structures [101, 102, 104 - 106].

In order to increase the light intensity absorbed in graphene $\mathrm{Xu}$ et al. have used optical interference in the underlying $\mathrm{SiO}_{2}$ layer [103]. They successfully detected electron injection from the top semitransparent electrode stack (Ti-Pt/ $/ \mathrm{Al}_{2} \mathrm{O}_{3} /$ graphene or $\mathrm{Ti}-\mathrm{Pt} / \mathrm{HfO}_{2} /$ graphene) with spectral threshold close to $3.3 \mathrm{eV}$ which has been ascribed to the energy barrier between the graphene Fermi level and the 
bottom of the $\mathrm{SiO}_{2} \mathrm{CB}$. Using the $\mathrm{SiO}_{2}$ electron affinity of $\chi\left(\mathrm{SiO}_{2}\right)=0.9 \mathrm{eV}$ this would yield a graphene $\mathrm{EWF}$ on $\mathrm{SiO}_{2}$ of $4.2 \mathrm{eV}$, suggesting n-type doping as opposed to the results inferred for the uncapped graphene on the basis of the builtin potential analysis by IPE [101] or using electrical measurements [107 - 110]. The origin of the n-doping of graphene upon encapsulation with $\mathrm{Al}_{2} \mathrm{O}_{3}$ can be understood as related to carbon residuals in alumina since a similar effect has recently been observed upon atomic-layer deposition (ALD) of $\mathrm{Al}_{2} \mathrm{O}_{3}$ on other 2D materials, e.g., $\mathrm{MoS}_{2}$ [111]. However, it is unclear whether or not the same doping mechanism associated with carbon residuals in the ALD-grown oxide cap will be relevant to the $\mathrm{HfO}_{2}$ capping. In fact, the spectral thresholds in the $3.2-3.3 \mathrm{eV}$ range observed in Ref. 103 have also been reported for electron IPE from Pt films similarly, as in Ref. 103, evaporated by ebeam on top of ALD-grown $\mathrm{Al}_{2} \mathrm{O}_{3}$ and $\mathrm{HfO}_{2}$ [80]. It is, therefore, difficult even to conclude whether the observed photocurrent is due to electron IPE from graphene into $\mathrm{SiO}_{2}$ or, else, from the Pt top electrode into the $\mathrm{Al}_{2} \mathrm{O}_{3}$ interlayer. The IPE spectra are distorted by the process of optical interference used to enhance the light intensity, which prevents reliable determination of the exponent factor $p$ in the Powell's model function $\mathrm{Y} \sim\left(\mathrm{h} v-\Phi_{\mathrm{e}}\right)^{p}$ needed to detect differences expected to emerge given the dissimilarity in the DOS of initial electron states below the Fermi level in Pt $v s$. graphene. Obviously then it will be worth of attempting to observe electron IPE from the uncapped graphene under interference-free conditions.

The results of this attempt have recently been published [112] using large-area ( $\mathrm{cm}^{2}$ range) synthetic CVD-grown single layer graphene (SLG) sheet and extensive IPE signal averaging to reach the needed sensitivity. The graphene layer was transferred on top of a $50-\mathrm{nm}$ thick $\mathrm{SiO}_{2}$ film as described earlier to eliminate optical interference artifacts. Furthermore, three different non-transparent contact metals with significantly different WFs ( $\mathrm{Au}, \mathrm{Cu}, \mathrm{Al})$ were compared in order to exclude the contribution of the metal contact to the IPE current. Examples of IPE quantum yield spectra are shown in Figs. 4 and 5 insemi-logarithmic and $\mathrm{Y}^{1 / 3}$-hv (Powell plot) coordinates as measured under different incremental negative voltages applied to the nontransparent Au contact pad. The spectra are seen to follow well the Powell model behavior $\mathrm{Y} \sim\left(\mathrm{h} v-\Phi_{\mathrm{e}}\right)^{3}$ (cf. Fig. 5) which corresponds to the linear increase of the occupied electron DOS in the photoemitter [82] as expected for graphene below the Dirac point [113]. There is a clear fieldinduced shift of the IPE threshold to lower photon energies exposing the image-force barrier behavior. The spectra measured on samples with $\mathrm{Cu}$ and $\mathrm{Al}$ contact pads show similar behavior and photocurrent values indicating that the measured signal stems from electron IPE from the unmetallized graphene layer.

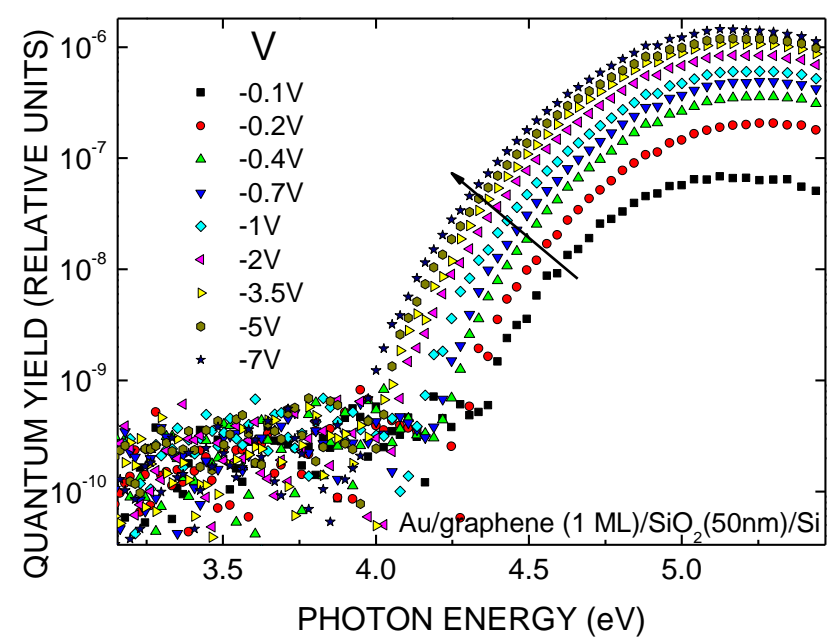

Figure 4. Semi-logarithmic plot of electron IPE quantum yield from single-ML graphene into $\mathrm{SiO}_{2}$ as measured under the indicated negative bias voltage $\mathrm{V}$ applied via a non-transparent $\mathrm{Au}$ contact pad.

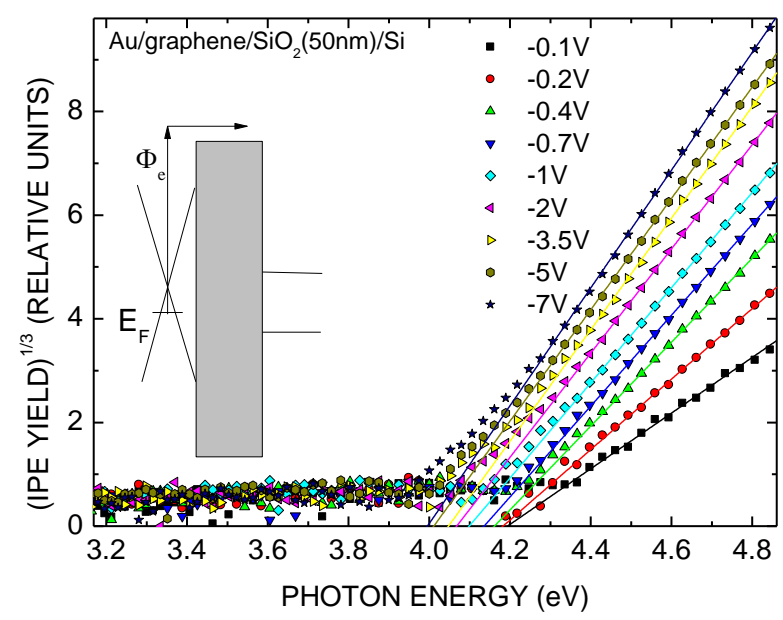

Figure 5. Powell plots of electron IPE quantum yield from singleML graphene into $\mathrm{SiO}_{2}$ as measured under the indicated negative bias applied via a non-transparent Au contact pad, illustrating the IPE threshold determination. The inset shows a schematic of the observed electron transitions. 


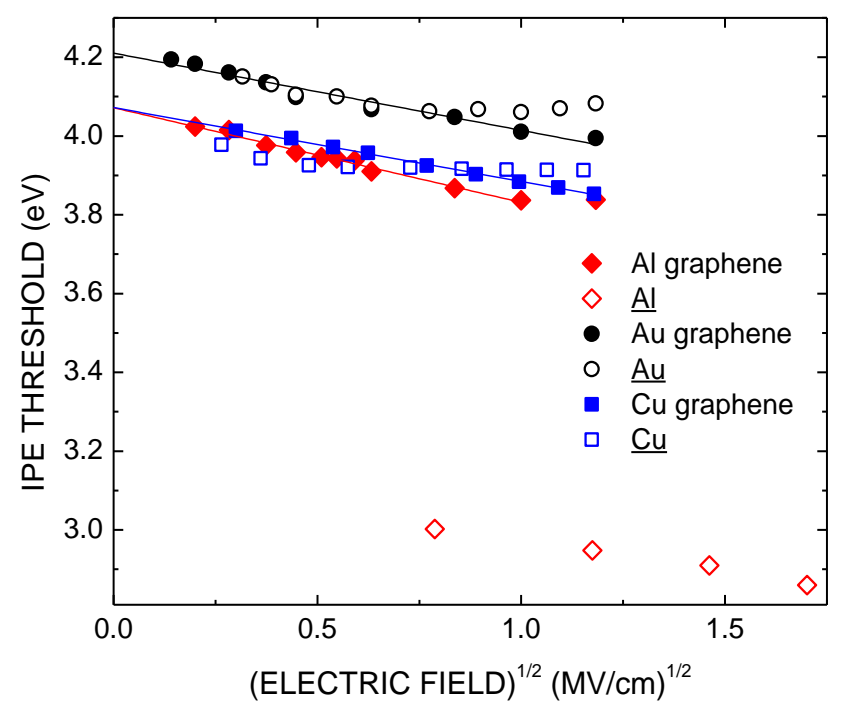

Figure 6. Schottky plots of electron IPE spectral thresholds from single-ML graphene (filled symbols) into $\mathrm{SiO}_{2}$ as measured on samples with different non-transparent metal contact pads $(\mathrm{Au}, \mathrm{Cu}$, Al). Open symbols show the thresholds of electron IPE from the semitransparent electrodes of the corresponding metals evaporated on the graphene-free part of the $\mathrm{SiO}_{2}$ surface. Lines, guiding the eye, illustrate the extrapolation to zero field.

To determine the barrier between the graphene Fermi level and the bottom of the $\mathrm{SiO}_{2} \mathrm{CB}$ the Schottky plots were made as exemplified in Fig. 6 for three different contact metals. Extrapolation to zero field yields the barrier of $\Phi_{\mathrm{e}}$ $=4.10 \pm 0.05 \mathrm{eV}$ for $\mathrm{Al}$ and $\mathrm{Cu}$ contacts and $\Phi_{\mathrm{e}}=4.20 \pm 0.05$ $\mathrm{eV}$ for the Au contact, pointing to the possibility of slight pdoping in the latter case. Most interesing, however, is the high value of EWF of 5-5.1 eV obtained by adding to the above barrier heights the value $\chi\left(\mathrm{SiO}_{2}\right)=0.9 \mathrm{eV}$. Though this value is approximately $0.5 \mathrm{eV}$ higher that the vacuum WF of graphene [114-116], it is well consistent with results of capacitance-voltage measurements in $\mathrm{Si} /$ oxide/graphene capacitors $[110,117,118]$ as well as with the values obtained by Kelvin probe microscopy [119] in the defoliated graphene case. It is worth of adding here that in the case of graphite-like amorphous carbon deposited directly on top of $\mathrm{SiO}_{2}$, the Fermi level is positioned at $3.6 \mathrm{eV}$ below the $\mathrm{SiO}_{2}$ CB bottom [120] yielding EWF $=4.5 \mathrm{eV}$, a value generally accepted both for graphite and undoped graphene. This observation might indicate that the transfer process affects graphene doping in that it causes a Fermi level shift. For example, the presence of water at the interface between $\mathrm{SiO}_{2}$ and graphene is known to cause hole doping of the latter [27]. It is also possible that the observed EWF variations reflect the presence of a polarization layer at the oxide surface which, in the case of van de Waals bonding, is prone to chemical interactions with the environment and with reagents involved in the graphene transfer.

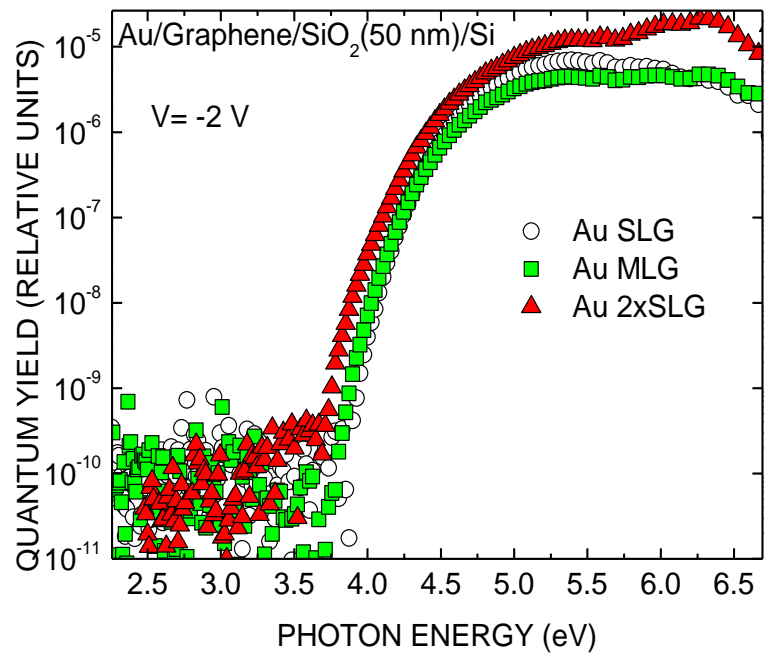

Figure 7. Semi-logarithmic plot of electron IPE quantum yield from SLG, MLG and $2 \times$ SLG graphene into $\mathrm{SiO}_{2}$ as measured under $-2 \mathrm{~V}$ bias applied via a non-transparent Au contact pad.

\subsection{Impact of graphene processing}

Since the CVD synthesis of graphene requires high temperatures $\left(>1000{ }^{\circ} \mathrm{C}\right)$, its direct growth onto $\mathrm{Si} / \mathrm{SiO}_{2}$ or $\mathrm{Si} / \mathrm{high}-\mathrm{k}$ oxide substrates will irrepairably damage the insulating layers leading to high electrical leakage that would make IPE current detection impossible. Therefore, all the measurements described in this work were performed using graphene transfer on top of $\mathrm{SiO}_{2}$ as described in section 4.5. Nevertheless, one can examine the impact of layer transfer on the interface barrier between graphene and the oxide by comparing IPE of electrons from multi-layer graphene (MLG) to that of two SLG sheets sequentially transferred onto $\mathrm{SiO}_{2}$.

The IPE yield spectral plots for the reference SLG sample, the MLG one, and the $2 \times$ SLG stack on top of $\mathrm{Si} / \mathrm{SiO}_{2}(50 \mathrm{~nm})$ substrate are compared in Fig. 7 as semilogarithmic spectral plots taken under the same bias of $-2 \mathrm{~V}$ applied to the graphene electrode to enable electron injection into $\mathrm{SiO}_{2}$. While the difference between SLG and MLG appears marginal, the sequential transfer of two SLG sheets leads to noticable red-shift of the IPE spectral onset. To get more insight into the reason for this shift, the field dependences of the IPE spectral thresholds have been addressed. As can be seen from the Schottky plots shown in Fig. 8, the field-induced barrier lowering exhibits the same trend for SLG and MLG with, however, a $\approx 70 \mathrm{meV}$ lower barrier for the latter. This might suggest that the barrier height variation is caused by a slight change of graphene doping rather than by a variation of the electrostatic potential distribution at the interface. The smaller barrier 
lowering than that predicted by the ideal image-force model for $\mathrm{SiO}_{2}$ (dashed black line in Fig. 8) points towards the presence of an additional electric field probably related to the presence of charged impurities remaining at the oxide surface after graphene transfer.

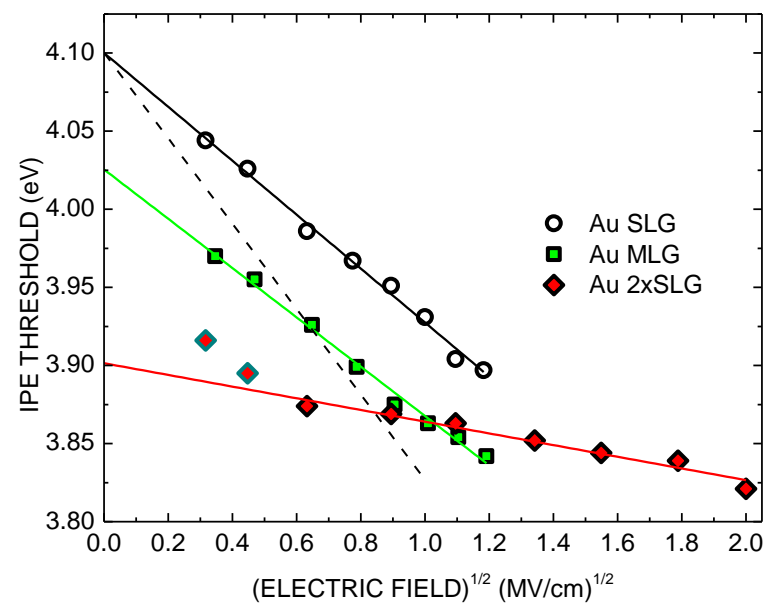

Figure 8. Schottky plots of electron IPE spectral thresholds from SLG, MLG, and $2 \times$ MLG samples into $\mathrm{SiO}_{2}$ as measured on samples with non-transparent Au contact pads. Lines guide the eye in the extrapolation to zero field. The dashed black line shows the barrier lowering expected for the ideal image-force barrier in $\mathrm{SiO}_{2}$.

By contrast, the $2 \times$ SLG sample shows a much weaker barrier lowering over an extended electric field range, suggesting perturbation of the barrier shape, with, moreover, threshold values measured at low field clearly not following the trend. The latter suggests the presence of additional electric field(s) at the interface. This would be consistent with violation of interface electroneutrality caused by incorporation of charged impurities at the interface or in the near-surface $\mathrm{SiO}_{2}$ region upon transfer of the second graphene layer and subsequent annealing. One of the hypothetical mechanisms might be the closure of discontinuities in the first-layer graphene electrode which imperfections would, otherwise, allow contaminants to escape during the post-transfer annealing. For example, water is known to intercalate interface between graphene and $\mathrm{SiO}_{2}$ [27], which, upon annealing, results in generation of oxide defects $[121,122]$.

\subsection{Graphene/metal heterojunctions}

If considering heterojunctions comprised of $2 \mathrm{D}$ graphene and $3 \mathrm{D}$ metals, the IPE methodology offers an interesing opportunity to determine the energy separation between the Fermi levels of two conductors. Below, we will present two examples demonstrating how this approach may help to reveal intricate details about the electronic structure of such heterostructures.
First, it is possible to deposit a thin (semitransparent) metal contact on top of the transferred graphene layer by thermoresistive evaporation in vacuum. In this case, the damage to the graphene layer is minimal because of the low energy (in order of the thermal energy) carried by the metal atoms arriving to the surface. For example, evaporation of a 13-nm thick Al layer onto SLG leaves the spectra of electron IPE of graphene nearly unchanged as illustrated in Fig. 9, suggesting that the graphene is not damaged and its Fermi level position is not affected significantly. The major effect of $\mathrm{Al}$ evaporation is seen as the appearance of a low-energy IPE band with energy onset at $h v \approx 3-3.1 \mathrm{eV}$. The latter is close to the electron IPE threshold at the $\mathrm{Al} / \mathrm{SiO}_{2}$ interface formed by $\mathrm{Al}$ evaporation on the graphene-free part of the $\mathrm{Si} / \mathrm{SiO}_{2}$ substrate, in good agreement with literature results [123]. This observation indicates that $\approx 1 \mathrm{eV}$ contact potential difference between $\mathrm{Al}$ and graphene is accomodated at the interface between these conductors without significant charge transfer to graphene as suggested by nearly unchanged Fermi level position. This behavior points towards the presence of an interlayer, e. g., organic residues [24] or oxidation of $\mathrm{Al}$ at the interface caused by water adsorbates [25] on the graphene surface. Since the field dependences of the IPE thresholds from $\mathrm{Al}$ and graphene, shown in Fig. 10 as Schottky plots, are close, both photoemitters must be in direct contact with $\mathrm{SiO}_{2}$, i.e., the observed IPE from $\mathrm{Al}$ is probably related to the metal in discontinuities (pores, cracks,...) of the transferred graphene sheet. Nevertheless, since the Al electrode represents an equipotential plane, the position of the Fermi level remains the same across the whole Al layer, including its interface with the graphene sheet.

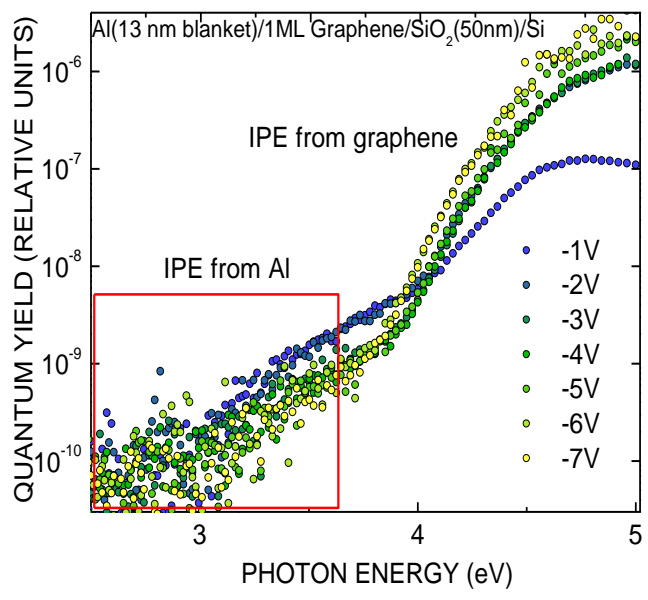

Figure 9. Semi-logarithmic plot of electron IPE quantum yield from a single-ML graphene sheet covered with a semitransparent (13 nm thick) $\mathrm{Al}$ overlayer into $\mathrm{SiO}_{2}$, as measured under the indicated negative bias applied to the metal electrode. 


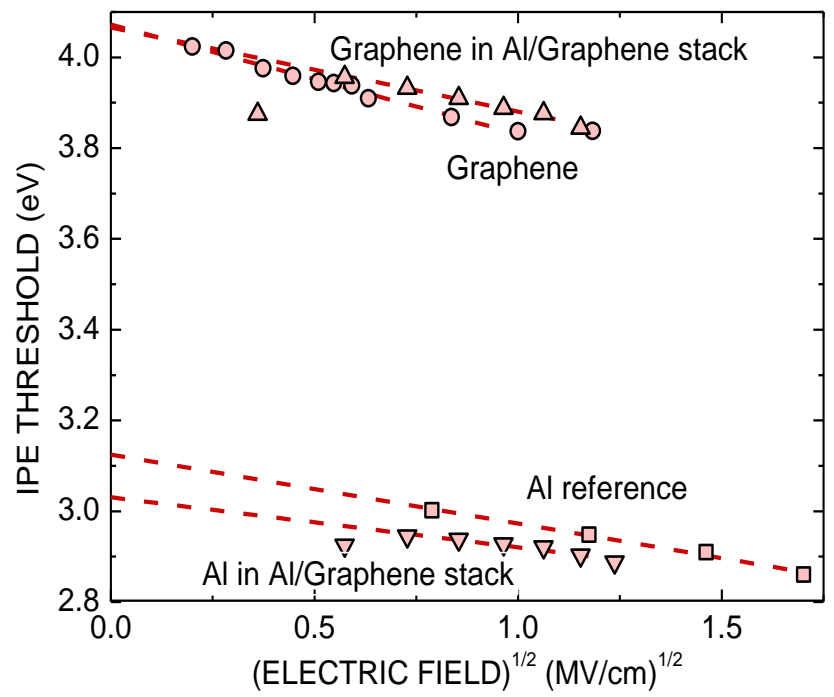

Figure 10. Schottky plots of electron IPE spectral thresholds from single-ML graphene with a semitarnsparent (13-nm thick) blanket Al electrode. For comparison, results are shown for the uncapped graphene sample and for the reference $\mathrm{Al}$ electrode evaporated onto the graphene-free $\mathrm{SiO}_{2}$ surface. Dashed lines guide the eye in the extrapolation to zero field.

An alternative possibility to study graphene/metal contacts using IPE can be realized by transfer of graphene onto pre-patterned metal electrodes on top of the insulating layer. As an example, Fig. 11 shows electron IPE spectra from the SLG (a) and MLG (b) transferred on top of 3-nm thick $\mathrm{Ru}$ electrodes deposited on a $50-\mathrm{nm}$ thick $\mathrm{SiO}_{2}$ film. The presence of $\mathrm{Ru}$ is clearly seen to provide a low-energy IPE onset while IPE from graphene is observed to become dominant in the spectral range $h v>4 \mathrm{eV}$. For both SLG and MLG the barrier between the Fermi level and the $\mathrm{SiO}_{2} \mathrm{CB}$ bottom remains in the range $4.05-4.10 \mathrm{eV}$, suggesting that its p-type doping is barely affected as compared to the earlier discussed case of uncapped graphene. The barrier height between the Fermi level of Ru and the oxide CB is slightly lower, in the range $3.7-3.8 \mathrm{eV}$, which corresponds to the EWF of $\mathrm{Ru}$ deposited onto $\mathrm{SiO}_{2}$ of 4.6-4.7 eV, i.e., significantly lower than the recommended vacuum WF values, typically exceeding $5 \mathrm{eV}$ [124]. This example importantly shows that at the real Ru/graphene interfaces, the Fermi level of graphene is energetically positioned well below that in the metal whereas the inverted positioning would be expected if using vacuum WF values. Obviously then, the use of vacuum WFs to evaluate contact potential differences at the real interfaces should be disencouraged. At the same time, the IPE metrology provides the possibility to measure the real barrier heights directly.
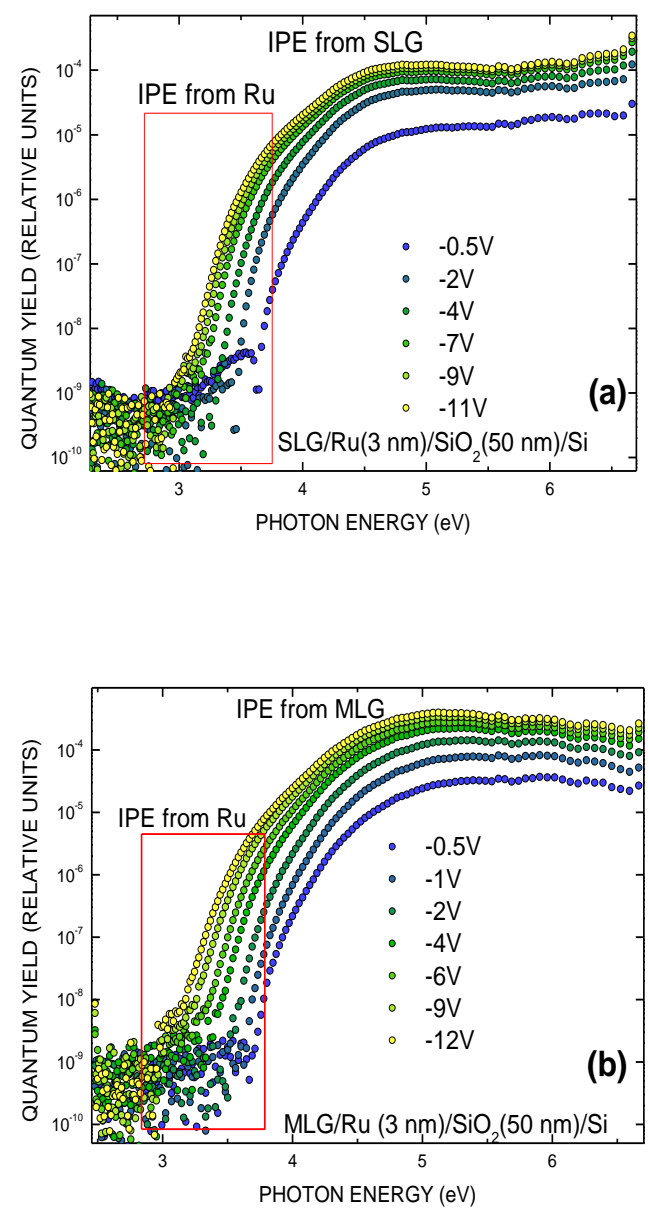

Figure 11. Semi-logarithmic plot of electron IPE quantum yield from SLG (a) and MLG (b) transferred on top of patterned 3-nm thick $\mathrm{Ru}$ electrodes deposited on a $50 \mathrm{~nm} \mathrm{SiO} 2$ layer, measured under the indicated negative voltages applied to the graphene/Ru stack.

\section{Interfaces of transition metal dichalcogenides}

Semiconductors of the TMD family are frequently considered as the prototype $2 \mathrm{D}$ materials suitable for the ultimate MISFET channel scaling down to the single ML thickness. In particular, some of these, e.g., $\mathrm{MoS}_{2}$ and $\mathrm{WS}_{2}$, are reasonably stable in air and in aqueous solutions even in the ML form, making them suitable for device fabrication. Furthemore, $\mathrm{MoS}_{2}$ is widely available from geological sources and, therefore, one- and few-ML layers can be obtained by the defolation method. As a result, a vast majority of experimental research is still focussed on these two sulphides and it thus seems logical to start the overview of IPE applications from the interfaces of these TMDs. 
6.1 IPE from the valence band of TMDs: Impact of metal cation and chalcogen type

Thanks to lower synthesis temperature involved, many TMDs can be directly grown on $\mathrm{Si} / \mathrm{SiO}_{2}$ substrates, thus allowing one to avoid possible uncertainties related to poor purity control in the process of layer exfoliation and transfer. As a result one may compare IPE from TMD layers of different thickness and composition. As an example of such comparison [86], Figs. 12 and 13 show IPE spectra from few-ML $\mathrm{MoS}_{2}$ and $\mathrm{WS}_{2}$ layers grown using sulfurization of a thin film of the corresponding metal in $\mathrm{H}_{2} \mathrm{~S}$ at $800{ }^{\circ} \mathrm{C}$ [96]. The spectra [cf. panels (b)] are seen to follow Powell's power law $\mathrm{Y} \sim\left(\mathrm{h} \nu-\Phi_{\mathrm{e}}\right)^{3}$ in the photon energy range $\approx 1 \mathrm{eV}$ above the spectral threshold of photoemission, which is consistent with the linear increase of the electron DOS in the VB of $\mathrm{MoS}_{2}$ and $\mathrm{WS}_{2}$ observed in the external photoemission spectra $[83,84]$. This behavior leaves no doubt that the observed electron injection into $\mathrm{SiO}_{2}$ pertains to IPE from the occupied states of the TMD's VB [86 - 88].

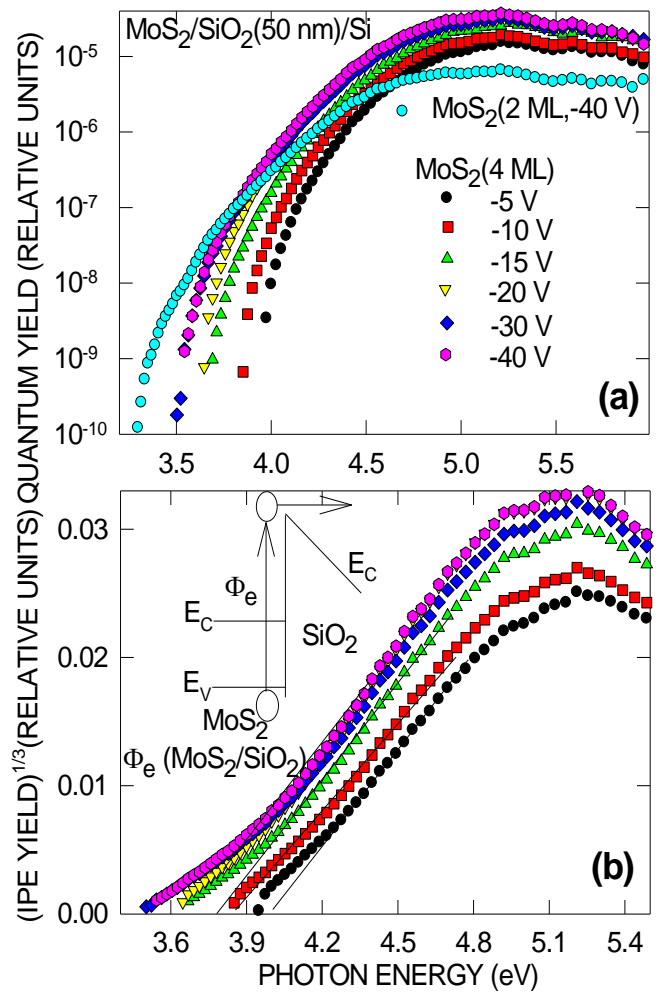

Figure 12. Semi-logarithmic (a) and Powell (b) plots of electron IPE quantum yield from few-ML $\mathrm{MoS}_{2}$ into $\mathrm{SiO}_{2}$ as measured under the indicated negative bias applied via a non-transparent $\mathrm{Au}$ contact pad. The inset in panel (b) shows a schematic of the observed electron transitions.

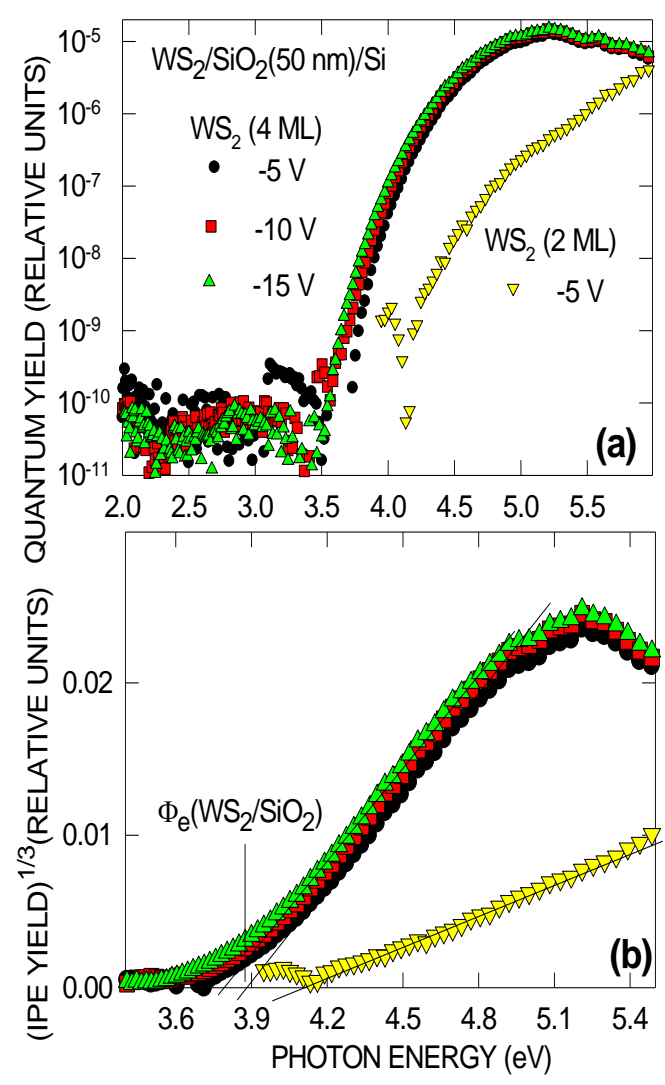

Figure 13. Semi-logarithmic (a) and Powell (b) plots of electron IPE quantum yield from few-ML $\mathrm{WS}_{2}$ into $\mathrm{SiO}_{2}$ as measured under the indicated negative bias applied via a non-transparent $\mathrm{Au}$ contact.

The spectral thresholds of IPE from the VBs of $\mathrm{MoS}_{2}$ and $\mathrm{WS}_{2}$ are close in energy, as can be seen from the spectra directly compared in Fig. 14(a), although the different lowenergy "tails" of the curves suggest the presence of gap states with a different density. The spectral thresholds are also found to be field-dependent (cf. Figs. 12 and 13). If applying the Schottky model for the image-force barrier lowering, the zero-field barriers in the range 4.0-4.2 eV are typically obtained for the both sulfurization-grown $\mathrm{MoS}_{2}$ and $\mathrm{WS}_{2}$ as can be seen from the Schottky plot shown in Fig. 14 (b). This observation suggests that the VB top energy in these semiconductors is marginally sensitive to the cation type, i.e., the band alignment follows the so-called "common anion rule" referring to the S3p states dominating the electron DOS in this energy range. Thus, the behavior of interface barriers for the semiconducting sulfides looks similar to that of the earlier studied insulating metal oxides [30] where the VB top is derived from the lone-pair O2p states, the latter remaining at the same energy $(\approx 2.5 \mathrm{eV}$ 
below the silicon VB top) for cations with sufficiently large ionic radii.

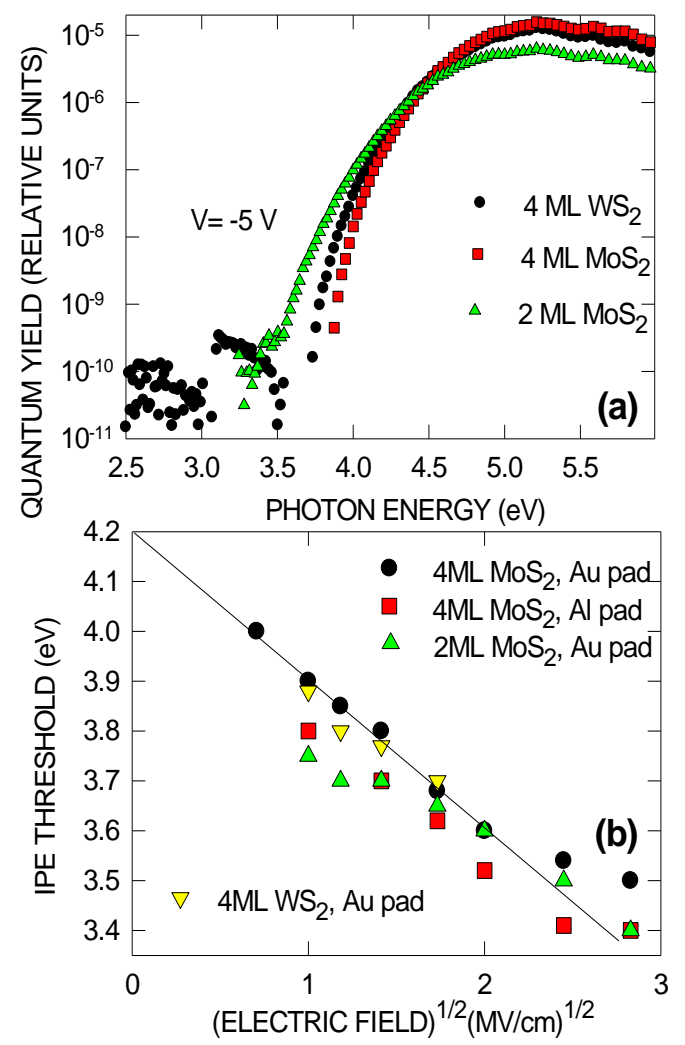

Figure 14. (a) Comparison of IPE spectra measured under $-5 \mathrm{~V}$ bias applied via a non-transparent $\mathrm{Au}$ contact pad to $\mathrm{MoS}_{2}$ and $\mathrm{WS}_{2}$ layers of different thicknesses. (b) Schottky plot of spectral thresholds of electron IPE from different $\mathrm{MoS}_{2}$ and $\mathrm{WS}_{2}$ electrodes into $\mathrm{SiO}_{2}$ with different contact pad metals.

Though the described behavior of the $\mathrm{MoS}_{2} / \mathrm{SiO}_{2}$ and $\mathrm{WS}_{2} / \mathrm{SiO}_{2}$ barriers follows, at least at first sight, the pattern known earlier for 3D semiconductor/insulator interfaces with chemical bonding, there seems to be an unexpectedly high sensitivity of the measured barrier heights to the chemistry of TMD synthesis. A comparison between IPE spectra [57] from the $\mathrm{MoS}_{2}$ layers grown in the H- and Cfree environment [100] and the above discussed metal sulfurization method [96] reveals a $\approx 0.5 \mathrm{eV}$ interface barrier difference. Since a VB top position, very closevery close to that of the sulfurization $\mathrm{MoS}_{2}$ case, is also found in the films synthesized by thermal sulfurization of $\mathrm{MoO}_{3}$ in $\mathrm{H}_{2} \mathrm{~S}$ [99] or in the layers grown by $\mathrm{CVD}$ using $\mathrm{Mo}(\mathrm{CO})_{6}$ and $\mathrm{H}_{2} \mathrm{~S}$ precursors [98], it was hypothesized that the barrier variability is caused by the presence of hydrogen during TMD synthesis. Since the slope of the Schottky plot remains unchanged within the measurement accuracy, hydrogen probably interacts with the surface of $\mathrm{SiO}_{2}$ with the formation of a dipole as a result [57]. Worth of mentioning here is that CVD growth of $\mathrm{WS}_{2}$ using $\mathrm{W}(\mathrm{CO})_{6}$ and $\mathrm{H}_{2} \mathrm{~S}$ precursors [98] on top of $\mathrm{SiO}_{2}$ also leads to a somewhat lower electron barrier height, of about $3.7 \mathrm{eV}$, as compared to the $\approx 4 \mathrm{eV}$ barrier for the sulfurization-synthesized $\mathrm{WS}_{2}$ (cf. Fig. 14). This might suggest that variations of the interface barrier may also be affected by the TMD overlayer composition. From these results we may conclude that, as opposed to the chemically bonded interfaces, in the case of van de Waals bonded interfaces between TMD and $\mathrm{SiO}_{2}$ one can expect processing-sensitive deviations from the Schottky-Mott behavior of the order of $0.5 \mathrm{eV}$.

Next, there is a fact that the barrier height for electrons at the $\mathrm{MoS}_{2} / \mathrm{SiO}_{2}$ interface becomes sensitive to the thickness of the $\mathrm{MoS}_{2}$ layer when approaching the single-ML limit due to bandgap widening. Most of the gap opening is seen to occur at the VB side [57] suggesting a $\approx 0.4 \mathrm{eV}$ downshift in energy of the S3p-derived states near the VB top. Then, the presence of islands of the $2^{\text {nd }}$ and $3^{\text {rd }}$ MLs on top of the single ML $\mathrm{MoS}_{2}$ [98] will lead to lateral variations in the VB top energy position and, consequently, impair the hole transport properties of the TMD semiconductor film.

Furthermore, violation of the Schottky-Mott rule is clearly revealed when comparing electron IPE from $\mathrm{MoS}_{2}$ into $\mathrm{SiO}_{2}$ with IPE into $\mathrm{Al}_{2} \mathrm{O}_{3}$ using the VB top of the substrate $\mathrm{Si}$ crystal as the common energy reference [87]: The thresholds for electron IPE from the $\mathrm{Si}$ VB into $\mathrm{SiO}_{2}$ $(4.25 \mathrm{eV})$ and into $\mathrm{ALD}$-grown amorphous $\mathrm{Al}_{2} \mathrm{O}_{3}(3.25 \mathrm{eV})$ differ, pointing to $\mathrm{a} \approx 1 \mathrm{eV}$ difference in the electron affinities of these oxides. However, the IPE threshold from a single-ML $\mathrm{MoS}_{2}$ appears to be barely influenced when changing the oxide from $\mathrm{SiO}_{2}$ to $\mathrm{Al}_{2} \mathrm{O}_{3}$, indicating that the intrinsic differences in the bulk electron structure of the distinct insulating oxide are "compensated" by a dipole (or charges) at the van der Waals bonded $\mathrm{MoS}_{2} /$ oxide interface.

Finally, since the electron states near the VB top in TMD materials are derived from the electron states of anion atoms, it may be worthwhile to compare the studied TMD sulfides with TMDs containing other chalcogen atoms. As an example, Fig. 15 compares spectra of electron IPE from the VB of 4-ML thick $\mathrm{WS}_{2}$ and 4-ML thick $\mathrm{WSe}_{2}$ synthesized by sulfurization and selenization, respectively, of metallic $\mathrm{W}$ films evaporated on $\mathrm{SiO}_{2}$. The observed $\sim 0.6$ $\mathrm{eV}$ inctrease in the electron IPE spectral thresholds corresponds to a downshift in energy of the Se-derived VB top edge in $\mathrm{WSe}_{2}$ as compared to the $\mathrm{WS}_{2}$ layer. This conclusion comes as a surprise since theoretical calculations predict that the VB top in $\mathrm{WSe}_{2}$ lies energetically $\approx 0.6 \mathrm{eV}$ higher than that in $\mathrm{WS}_{2}[125,126]$. In fact the same trend, 
i.e., the VB of a selenide lying energetically deeper than that of sulfide, can be inferred from combining the recent results of IPE analysis of the $\mathrm{MoSe}_{2} / \mathrm{SiO}_{2}$ interface [127] to the data shown in Figs. 12 and 15: The observed barrier of $4.8 \mathrm{eV}$ between the VB top in $\mathrm{WSe}_{2}$ and the $\mathrm{SiO}_{2} \mathrm{CB}$ bottom [127] would place the $\mathrm{Se} 4 \mathrm{p}$ states at $\sim 0.6 \mathrm{eV}$ below the $\mathrm{S} 3 \mathrm{p}$ derved $\mathrm{MoS}_{2} \mathrm{VB}$ top energetically positioned at $4.2 \mathrm{eV}$ below the $\mathrm{SiO}_{2} \mathrm{CB}$ bottom (cf. Fig. 14). The only concern here is the use of the $\mathrm{MoSe}_{2}$ layer transfer on top of the insulating oxide which, as will be shown below, may have a significant impact on the measured barrier height. Nevertheless, the energy position of the VB at the interfaces of $\mathrm{MoSe}_{2}$ transferred on top of ALD-grown $\mathrm{Al}_{2} \mathrm{O}_{3}\left(\Phi_{\mathrm{e}}=4.10\right.$ $\mathrm{eV}$, Ref. 127) appears to be not far from than in multilayer $\mathrm{SnSe}_{2}$ with $\mathrm{Al}_{2} \mathrm{O}_{3}$ deposited on top $(\approx 4.4 \mathrm{eV}$ as can be estimated from Fig. 3 in Ref. 128) suggesting validity of the 'common anion rule' for selenides.
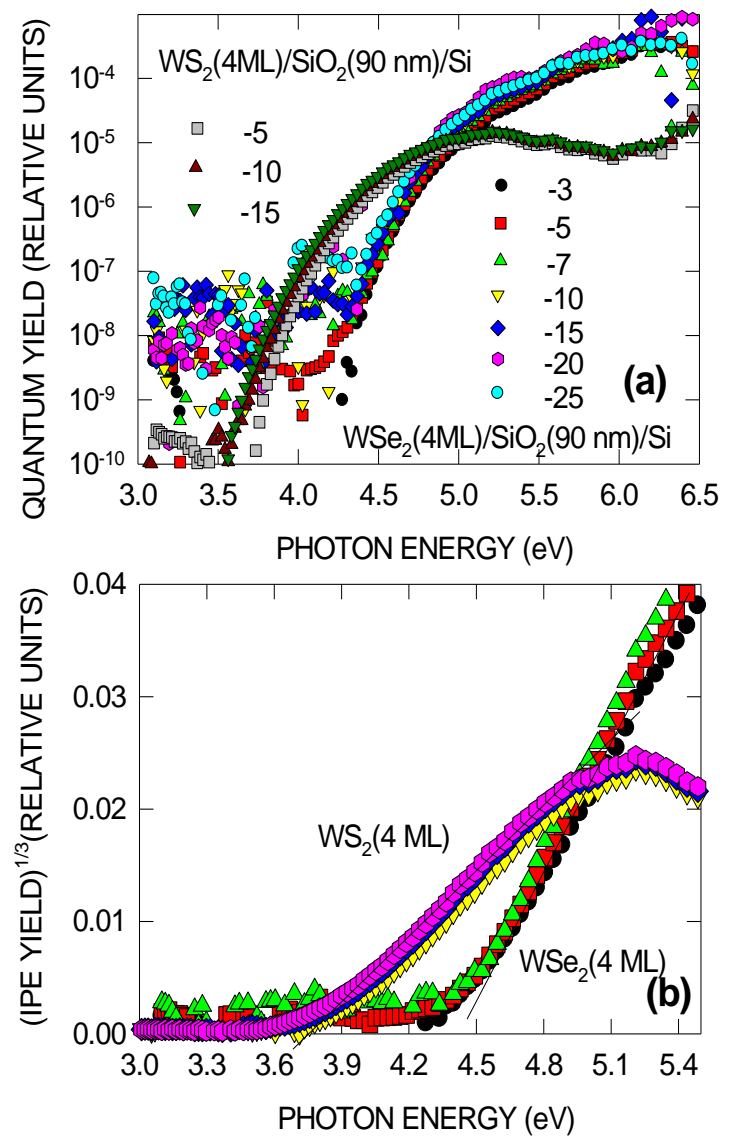

Figure 15. Semi-logarithmic (a) and Powell (b) plots comparing spectra of electron IPE spectra from 4-ML thick $\mathrm{WS}_{2}$ and $\mathrm{WSe}_{2}$ into $\mathrm{SiO}_{2}$ under the indicated bias applied via a non-transparent $\mathrm{Au}$ contact pad.

\subsection{Impact of TMD interface processing}

As already mentioned previously, in the course of device fabrication involving TMD semiconductors, the latter can be exposed to different ambients and reagents which, taking into account their ML-range thickness, may have a profound effect on electronic properties, including interface band alignment. In particular, the layer defoliation and transfer widely used to combine dissimilar 2D materials in functional heterostructures may lead to significant nonidealities [28, 86]. The detrimental effect of the transfer process may be caused both by modification of the electron states in the 2D layer as well as by "formation" of a van der Waals interface with another material. Therefore, to estimate the band alignment variations caused by the transfer processing, we first addressed the stability of 2D sample layers, $\mathrm{MoS}_{2}$ and $\mathrm{WS}_{2}$, under different environmental conditions.

While the sulfurization-grown $\mathrm{MoS}_{2}$ layers (2- and 4-ML thick) appear sufficiently stable under ambient conditions (at least over a period of 6 months [86]), similarly synthesized 2-ML $\mathrm{WS}_{2}$ films show gradual degradation resulting in a reduction of the IPE yield with time as illustrated in Fig. 16. The decrease of the IPE yield points toward a reduced electron DOS available in the $\mathrm{WS}_{2}$ layer, which is probably caused by its gradual oxidation. The presence of adsorbate layers on solid surfaces is well known [129, 130]; an observed density close to $1 \mathrm{~g} / \mathrm{cm}^{3}$ indicates water to be its major constituent [25]. Further experimental evidence for the oxidation-induced attenuation of the IPE yield came from a study of the effects of UV-ozone surface treatment [131], both for $\mathrm{MoS}_{2}$ and $\mathrm{WS}_{2}$ films. Quite surprisingly, the single-ML $\mathrm{WS}_{2}$ grown by CVD from $\mathrm{W}(\mathrm{CO})_{6}$ and $\mathrm{H}_{2} \mathrm{~S}$ precursors exhibit a much better stability against oxidation in air with detectable IPE signals still present even after 1 year storage.

Since $\mathrm{MoS}_{2}$ emerges now as the most stable 2D film, the effect of defoliation and layer transfer on the band alignment with $\mathrm{SiO}_{2}$ has been explored by comparing electron IPE at the 2-ML $\mathrm{MoS}_{2} / \mathrm{SiO}_{2}$ interfaces prepared by direct growth (metal sulfurization) and by transfer of the identically synthesized $\mathrm{MoS}_{2}$ on top of the $\mathrm{SiO}_{2}$ layer $[28,86]$. The IPE yield spectra shown in Fig. 17 reveal transfer-induced "blueshift" of the spectral thresholds by $\approx 1 \mathrm{eV}$, with attendant attenuation of their field dependence. Weak barrier lowering and the barrier height increase indicate incorporation of negative charges and/or dipoles, probably associated with well known water-oxide interactions leading to the formation of silanol groups and electron trapping [71, 72, $121,122,133,134]$. The interface electroneutrality can partially be restored by applying post-transfer annealing in $\mathrm{H}_{2} \mathrm{~S}$ [28]. However, such annealing cannot be universally 
used because of the highly corrosive nature of gaseous $\mathrm{H}_{2} \mathrm{~S}$. Summarizing these results, one may conclude that the processing-induced band alignment variations have a profound impact - of the order of $0.5-1 \mathrm{eV}$ - and can be seen as one of the most significant factors limiting the applicability of the Schottky-Mott rule to the van der Waals bonded interfaces of 2D materials.
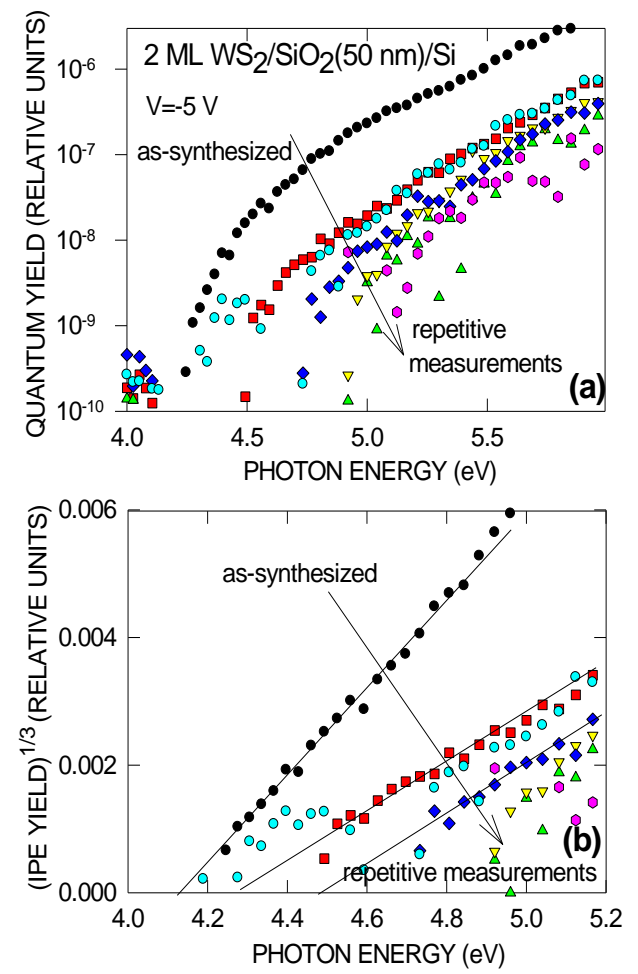

Figure 16. Semi-logarithmic (a) and Powell (b) plots illustrating the impact of storage in room air for a period of 6 months on repetitively measured IPE spectra from a $2-\mathrm{ML}$ thick $\mathrm{WS}_{2}$ sheet.

\subsection{TMD heterojunctions and contacts to metals}

The IPE experiments described above reveal a significant sensitivity of interface barriers and band alignment to the synthesis route and further processing of 2D TMDs, at least for interfaces of 2D sulfide semiconductors. It is obvious that the validity of the band offset transitivity principle generically related to the Schottky-Mott picture $[59,60]$ can be questioned. Nevertheless, if omitting cases of chemical modification of the TMDs, such as through oxidation, one may notice that the observed violations of interface electroneutrality are predominantly caused by the oxide substrate surface contributions. Therefore, it is instructive to examine band alignment at heterojunctions of two different 2D TMDs.

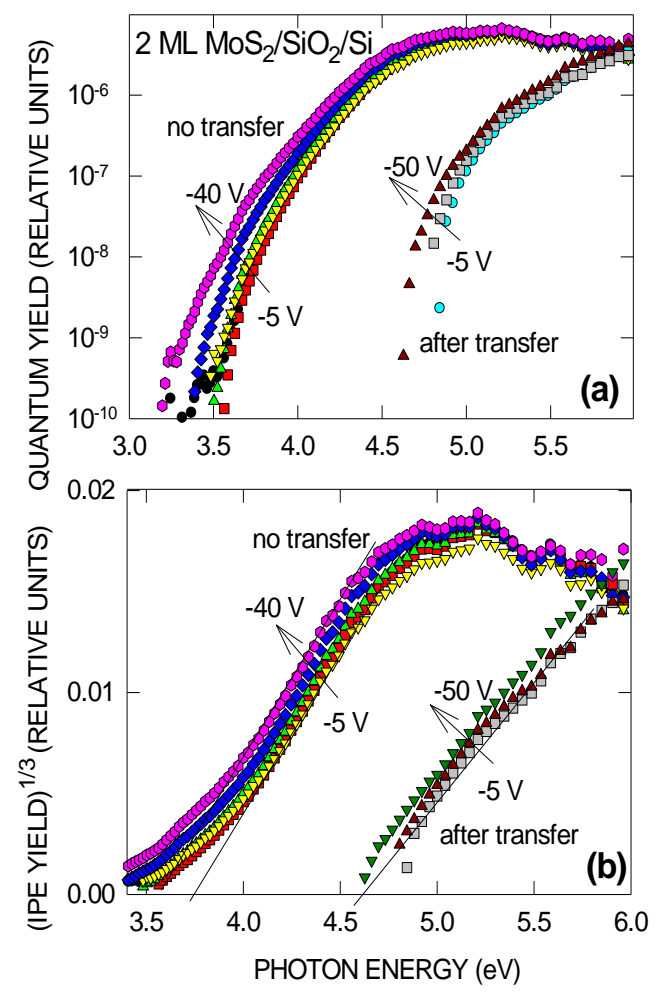

Figure 17. Semi-logarithmic (a) and Powell (b) plots illustrting impact of wet layer transfer on IPE spectra from a 2-ML thick $\mathrm{MoS}_{2}$ sheet.

These experiments [86] were conducted on samples prepared using the metal sulfurization method, which allows one not only to grow the few-ML thin TMD sheets directly on top of $\mathrm{SiO}_{2}$ but, also, to synthesize $\mathrm{MoS}_{2} / \mathrm{WS}_{2}$ and $\mathrm{WS}_{2} / \mathrm{MoS}_{2}$ stacks [96]: After completing the sulfurization anneal of the metal first evaporated onto the $\mathrm{SiO}_{2}$, another metal was deposited on top of the just formed TMD layer and subsequently followed by the second sulfurization step. Representative electron IPE spectra from such stacked electrodes are shown in Fig. 18. It is observed that neither the stacking order of $\mathrm{MoS}_{2}$ and $\mathrm{WS}_{2}$ layers nor their thickness (4 ML or $2 \mathrm{ML}$ ) affect the spectral thresholds within the accuracy limit of about $0.1 \mathrm{eV}$. Besides supporting the above conclusion regarding weak sensitivity of the VB top energy to the cation type, this observation further shows that the total thickness of the TMD film, reaching $8 \mathrm{ML}$, also has only a marginal effect on the IPE spectral thresholds. The thresholds remain virtually unchanged at low bias, but in higher fields the stacked electrodes exhibit significant low energy "tails". This effect can be associated with penetration of the electric field into the semiconducting electrode [31], leading to a measurable 
electrostatic potential drop inside the TMD film. As a result, electrons photoexcited in the TMD at some distance from the $\mathrm{TMD} / \mathrm{SiO}_{2}$ interface will "see" a lower energy barrier, leading to the experimentally observed IPE at lower photon energies. The important conclusion which can be drawn from this experiment is that the $\mathrm{VBs}$ of $\mathrm{MoS}_{2}$ and $\mathrm{WS}_{2}$ remain (nearly) aligned in the $\mathrm{WS}_{2} / \mathrm{MoS}_{2}$ heterojunction with no measurable interface dipole. This result obtained for the directly grown heterojunction is obviously different from the junctions formed by the exfoliation and transfer processing [135] underlining the need for reliable band offset quantification. IPE appears to provide one with a methodology to solve this problem.
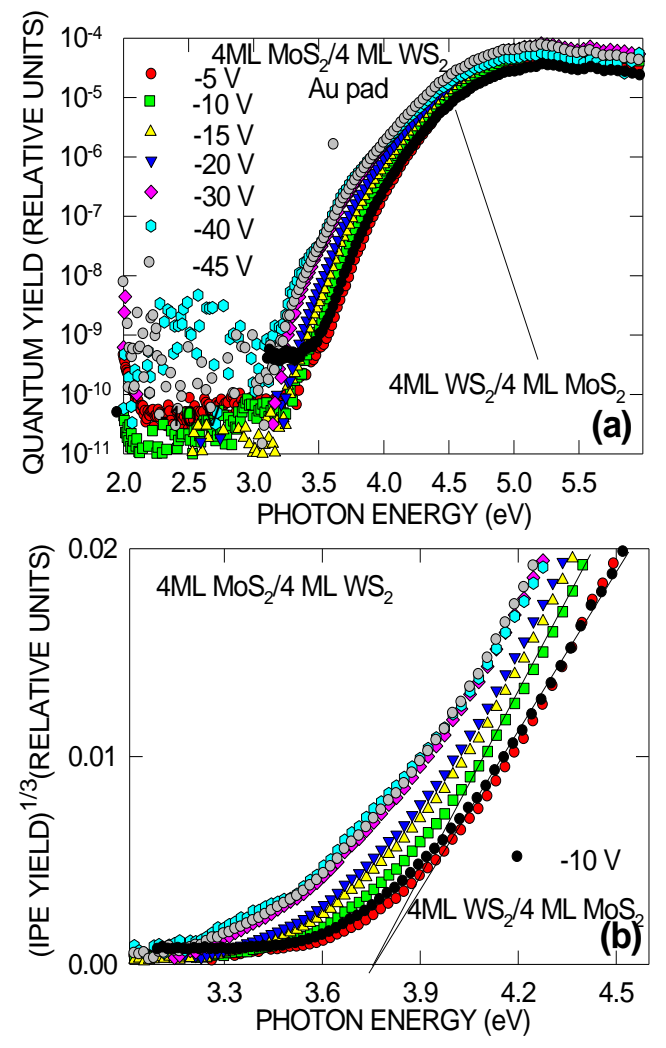

Figure 18. Semi-logarithmic (a) and Powell (b) plots comparing IPE spectra from $\mathrm{MoS}_{2} / \mathrm{WS}_{2}$ and $\mathrm{WS}_{2} / \mathrm{MoS}_{2}$ stacked electrodes into $\mathrm{SiO}_{2}$ under the indicated bias voltages applied via a nontransparent Au contact pad.

In a similar approach one may attempt to characterize metal/2D semiconductors contacts by observing onsets of electron IPE from the electron states near the Fermi level of the metal and from the semiconductor VB. This approach is exemplified in Fig. 19 showing a Schottky plot of electron IPE thresholds observed in a $1 \mathrm{ML} \mathrm{MoS}_{2} / \mathrm{Al}(15 \mathrm{~nm})$ stack on top of a 50 nm-thick $\mathrm{SiO}_{2}$ insulator; The $\mathrm{MoS}_{2} / \mathrm{Al}$ stack was fabricated by thermoresistive evaporation of large area $\left(0.5 \mathrm{~mm}^{2}\right)$ semitransparent $\mathrm{Al}$ electrode on top of $1 \mathrm{ML}$ $\mathrm{MoS}_{2}$ formed by magnetron sputtering of Mo in sulfur vapor [100].

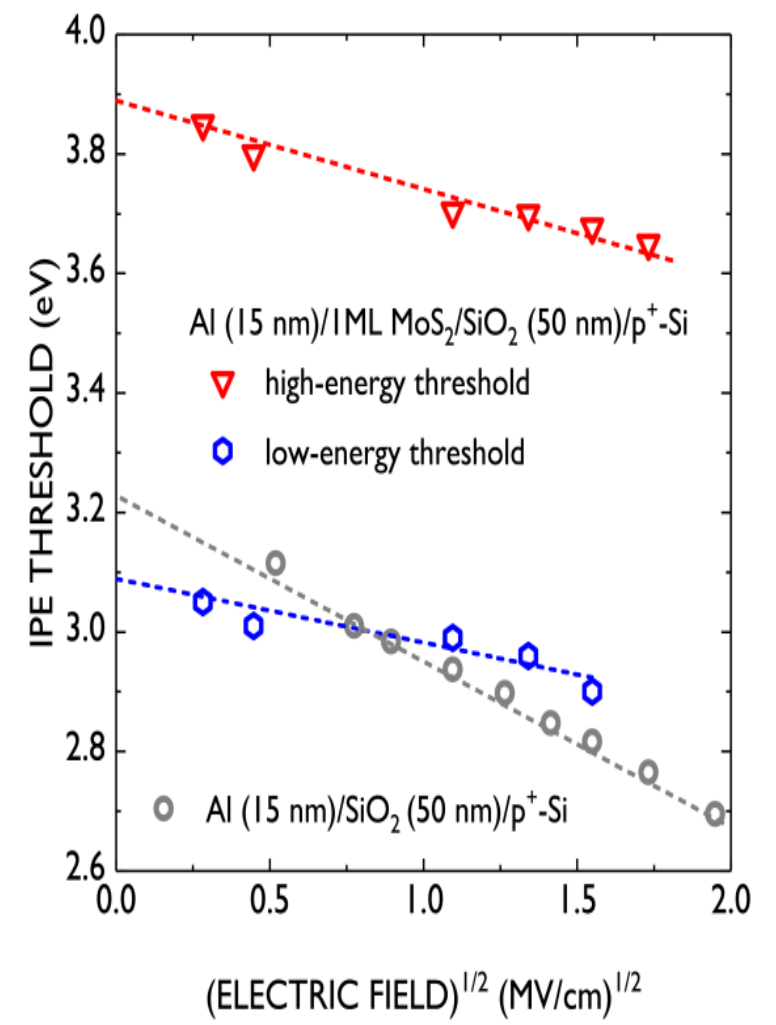

Figure 19. Schottky plot of the thresholds of electron IPE from the $\mathrm{Al}(15 \mathrm{~nm}) / \mathrm{MoS}_{2}(1 \mathrm{ML})$ electrode into $\mathrm{SiO}_{2}$ compared with that obtained for the case of a 15-nm thick Al layer evaporated onto $\mathrm{SiO}_{2}$ surface not covered by an $\mathrm{MoS}_{2}$ layer. Lines illustrate extrapolation to zero electric field strength.

The IPE spectral curves from this $\mathrm{MoS}_{2} / \mathrm{Al}$ contact stack show two spectral photoemission bands, one corresponding to the IPE of electrons from the $\mathrm{MoS}_{2} \mathrm{VB}$ and another, much attenuated and with significantly lower spectral threshold corresponding to the IPE of electrons from Al. For reasons of comparison, in Fig. 19 also shown are spectral thresholds of IPE from a similar 15-nm thick Al electrode evaporated on top of an un-processed $\left(\mathrm{MoS}_{2}\right.$-free) $\mathrm{Si} / \mathrm{SiO}_{2}$ substrate. The lowest threshold appears to be close to that of Al which allows us to associate it with IPE of electrons from the $\mathrm{Al}$ contact into $\mathrm{SiO}_{2}$. The change in the Schottky plot slope between two curves corresponding to the IPE from Al is probably caused by different screening geometry in which the $\mathrm{Al}$ photoemitter is separated from $\mathrm{SiO}_{2}$ by the $\mathrm{MoS}_{2}$ layer and by a thin film of $\mathrm{Al}$ oxide formed on top of $\mathrm{MoS}_{2}$ due to adsorbed water [25] as detected by transmission 
electron microscopy [136]. By comparing zero-field spectral threshold values, one can directly infer the $0.8 \mathrm{eV}$ high energy barrier between the $\mathrm{MoS}_{2} \mathrm{VB}$ top and the Fermi level of Al. Now, to complete the band diagram of the $\mathrm{MoS}_{2} / \mathrm{Al}$ contact one needs to determine the semiconductor bandgap width.

\subsection{IPE to traps and excitonic spectra}

Measurement of photoconductivity (PC) spectra provides one of the most straightforward methods of semiconductor bandgap width determination. In the case of one- or few-ML 2D semiconductors, the PC spectra look very similar to the spectra of optical absorption and contain the same excitonic features $[137,138]$. There is a problem, however, with the detection of $\mathrm{PC}$ in the synthetic 2D films related to high surface recombination rate, which leads to rapid decrease of the PC current when the length of photoconductor increases. To reach the $2 \mathrm{D}$ photoconductor length in the range of microns or below one has to use lithographic processing with associated problems of chemical stability of the MLthin films and concomitant contamination, particularly if analysing defoliated and transferred layers.

To avoid these problems, it has recently been proposed to detect optical generation of electron and holes in 2D semiconductor electrodes through sensing the displacement photocurrent generated upon the trapping of charge carriers in the surface layer of the underlying insulator or at the interface of the 2D material, i.e., the "IPE to traps" [32]. These measurements can be done in the same capacitor structures used for IPE measurements simply by shifting the spectral range to the near-gap region of the $2 \mathrm{D}$ semiconductor. An example of the photocurrent yield spectra recorded in the low-energy range on a CVD-grown single-ML WS $\mathrm{WS}_{2}$ sample transferred onto a $\mathrm{SiO}_{2}(50 \mathrm{~nm}) / \mathrm{Si}$ target substrate is shown in Fig. 20. Positive or negative voltages of 7 or $10 \mathrm{~V}$ were applied to the $\mathrm{WS}_{2}$ layer via an optically non-transparent (100 nm thick) Al contact evaporated on top of the transferred $\mathrm{WS}_{2}$. The photocurrent spectra show three distinct peaks, A, B, and C positioned at energies perfectly reproducing the energies of exciton absorption lines known from the optical spectra [139, 140]. Furthermore, at $\mathrm{h} v \approx 3.4 \mathrm{eV}$, i.e., energetically right below the onsets of electron IPE from $\mathrm{WS}_{2}($ at $\mathrm{V}<0)$ or $\mathrm{Si}$ substrate (at $\mathrm{V}>0$ ) into $\mathrm{SiO}_{2}$, one also observes the fourth peak $\mathrm{D}$ usually not recorded in the optical measurements performed in the visible spectral range.

The opposite direction of photocurrents observed under opposite bias polarities suggests trapping of electrons $(\mathrm{V}<0)$ or holes $(\mathrm{V}>0)$ optically excited in $\mathrm{WS}_{2}$ by traps positioned in the vicinity of the $\mathrm{SiO}_{2} / \mathrm{WS}_{2}$ interface. Apparently, dissociation of excitons is facilitated by trapping of one of charge carriers while the differences in the photocurrent yield values reflect differences in the density and in-depth distribution of electron and hole traps. The yield shows a general trend to increase with applied bias voltage for both orientations of electric field suggesting that the carriers excited in the 2D layer tunnel to the traps since their energy is by far insufficient to surmount the potential barrier at the interface and to enter the $\mathrm{SiO}_{2} \mathrm{CB}$.

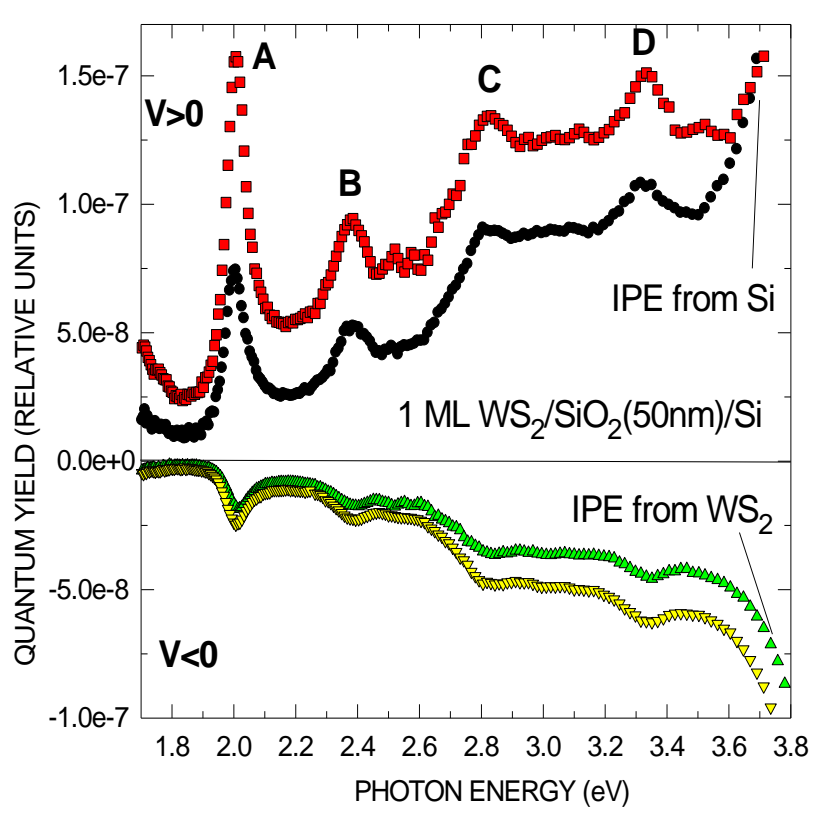

Figure 20. Photocurrent yield spectra observed in a $1 \mathrm{ML}$ $\mathrm{WS}_{2} / \mathrm{SiO}_{2}(50 \mathrm{~nm}) / \mathrm{Si}$ sample (dry transfer of $\mathrm{WS}_{2}$ ) under positive or negative bias voltages $(7$ or $10 \mathrm{~V})$ applied to the $\mathrm{WS}_{2}$ sheet via a non-transparent $\mathrm{Al}$ contact pad. Labels A-C indicate known excitonic peaks.

Very similar observations of excitons in the PC spectra were already reported for the one- and few-ML $\mathrm{MoS}_{2}$ layers directly grown or transferred onto $\mathrm{SiO}_{2}$ [32]. Two additional features make this mode of the PC measurements particularly interesting: First, since the PC is detected through charge carrier transfer across the interface and does not require lateral current flow, it can be detected even under a continuous semitransparent metal electrode which othewise would shunt the $2 \mathrm{D}$ semiconductor lateral PC. This allows one to analyze the impact of metallization of electron states in the $2 \mathrm{D}$ material as compare these to the unmetallized surface case illustrated above. For example, evaporation of $\mathrm{Cu}$ on top of $\mathrm{WS}_{2}$ leads to disappearance of the excitonic PC peaks, indicating a dramatic change of the electron states in the semiconductor upon metal evaporation 
and suggesting $\mathrm{Cu}$ intercalation. Second, since the lateral electrical conduction is not required to detect displacement currents in the capacitor structure, one can detect PC even in discontinuous 2D films on top of insulating substrate after evaporation of a semitransparent metal contact on top. The only condition here is that the insulating layer should be sufficiently thin to allow detection of displacement current generated by charge transfer from the optically ecxited 2D layer to near-interface traps. For traps located in $\mathrm{SiO}_{2}$ the electron tunneling distance is estimated to be about $2 \mathrm{~nm}$ $[141,142]$.

The next step in developing the "IPE to traps" as a reliable bandgap determination method should probably focus on separation of the excitonic transitions from the band-to-band transitions. This might be achieved by analyzing the field dependences of the excitonic and bandto-band PC since the large exciton binding energy in MLthin 2D semiconductors should lead to a significant difference in the initial energy of charge carriers tunneling to traps from an exciton state or from a band.

\section{Conclusions}

From the above overviewed initial results of applying IPE methods to the analysis of electron states at interfaces of 2D materials, we already can conclude that this experimental approach provides a significant amount of useful information regarding the energy distribution of electron states such as band offsets, barrier heights, bandgap energies as well as energies of elemental excitations. Most important, these results pertain to interfaces formed by using technologically relevant processing including defoliation and 2D layer transfer. Thanks to this, IPE can be used not only to characterize the intrinsic properties of 2D materials but, also, to monitor their behavior as affected by the environment and technological treatments.

What becomes already clear is that the ideal SchottkyMott picture appears to be insufficient to describe the band alignment at the interfaces of 2D materials with oxide insulators. Likely, more interfaces, dealing with different materials will be analyzed in the near future thanks to the rapid progress in development of wafer-scale 2D material synthesis. In any event, IPE seems to be firmly set for future analysis of low-dimensional heterojunctions.

\section{Acknowledgements}

The authors would like to express their deep gratitude to the members IMEC 2D-device team for the technological support which made this research possible: D. Chiappe, A. Leonhardt, S. Achra, S. Banerjee, G. Arutchelvan, J. Ludwig, S. El Kazzi, B. Groven, S. Brems, C. Hyughebaert, M. Caymax, S. de
Gendt, and I. Radu. We also gratefully acknowledge support of J. Chai, M. Yang, and S. J. Wang (A*STAR, Singapore), S. Monaghan and P.K. Hurley (Tyndall Institute, Ireland), A. Sharma and A. Bol (TU Eindhoven, the Netherlands), Ch. Martella and A. Molle (MDM Laboratories, Italy).

This work was financially supported by the Flanders Innovation \& Entrepreneurship [2Dfun (2D functional $\mathrm{MX}_{2}$ /graphene hetero-structures), an ERA-NET project in the framework of the EU Graphene Flagship] and by a KU Leuven Internal Fund (project C14/16/061).

\section{References}

[1] Terrones H, Lopez-Urias F and Terrones M 2013 Sci. Reports 31549

[2] Liu Y, Weiss N O, Duan X, Cheng H-C, Huang Y and Duan X 2016 Nature Rev. Mater. 11604

[3] Jena D 2013 Proc. IEEE 1011585

[4] Jena D, Banerjee K and Xing G H 2014 Nature Mater. 13 1076

[5] Yan R, Fathipour S, Han Y, Song B, Xiao S, Li M, Ma N, Protasenko V, Muller D A, Jena D and Xing G H 2015 Nano Lett. 155791

[6] Chhowalla M, Jena D and Zhang H 2016 Nature Rev. Mater. 1 16052

[7] Xu Y, Cheng C, Du S, Yang J, Yu B, Luo J, Yin W, Li E, Dong S, Ye P and Duan X 2016 ACS Nano 104895

[8] Radisavljevic B, Radenovic A, Brivio J, Giacometti V and Kis A 2011 Nature Nanotechnol. 6147

[9] McDonnell S, Azcatl A, Addou R, Gong C, Battaglia C, Chuang S, Cho K, Javey A and Wallace R M 2014 ACS Nano. 86265

[10] Ganatra R and Zhang Q 2014 ACS Nano 8, 4074

[11] Nourbakhsh A, Zubair A, Sajjad R N, Tavakkoli K G, Chen W, Fang S, Ling X, Kong J, Dresselhaus M S, Kaxiras E, Berggren K K, Antoniadis D and Palacios T 2016 Nano Lett. 167798

[12] Nourbakhsh A, Zubair A, Joglekar S, Dresselhaus M and Palacios T 2017 Nanoscale 186122

[13] Sarkar D, Xie X, Liu W, Cao W, Kang J, Gong Y, Kraemer S, Ajayan P M and Banerjee K 2015 Nature 52691

[14] Lyu J, Pei J, Gong J and Li H 2019 Adv. Mater. 1906000

[15] Chen H, Li J, Chen X, Zhang D and Zhou P 2018 Semicond. Sci. Technol. 33024001

[16] Ajayan P, Kim P and Banerjee K 2016 Physics Today 69(9) 38

[17] Li X, Tao L, Chen Z, Fang H, Li X, Wang X, Xu J-B and Zhu H 2017 Appl. Phys. Rev. 4021306

[18] Robinson J A 2018 APL Materials 6058202

[19] Lin Z, Lei Y, Subramanian S, Briggs N, Wang Y, Lo CL, Yalon E, Lloyd D, Wu S, Koski K, Clark R, Das S, Wallace R M, Kuech T, Bunch J S, Li X, Chen Z, Pop E, Crespi V H, Robinson J A and Terrones M 2018 APL Materials 6080701

[20] Donarelli M, Bisti F, Perrozzi F and Ottaviano L 2013 Chem. Phys. Lett. $\mathbf{5 8 8} 198$

[21] Bom N M, Soares G V, de Oliveira Jr M H, Lopes J M J, Riechert H and Radtke C 2016 J. Phys. Chem. C 120201

[22] Rolim G K, Correa S A, Galves L A, Lopes J M J, Soares G V and Radtke C 2016 Appl Surf Sci. 427825 
[23] McManus D, Vranic S, Withers F, Sanchez-Romaguera V, Macucci M, Yang H, Sorrentino R, Parvez K, Son S-K, Iannaccone G, Kostarelos K, Fiori G and Casiraghi C 2017 Nature Nanotechnol. 12, 343

[24] Haigh S J, Gholinia A, Jalil R, Romani S, Britnell L Elias D C, Novoselov K S, Ponomarenko L A, Geim A K and Gorbachev R 2012 Nature Mater. 11764

[25] Jiang L, Huang Q-S, Kozhevnikov I V, Yao Y-Y, Feng J-T, Feng H-F, Ma B, Jiao H-F, Chen H, Zhang Z and Wang ZS 2018 Chinese Phys. C 42115001

[26] Zheng C, Xu Z-Q, Zhang Q, Edmonds M T, Watanabe K, Taniguchi T, Bao Q and Fuhrer M S 2015 Nano Lett. 15 3096

[27] Koyama T, Inaba T, Komatsu K, Moriyama S, Shimizu M and Homma Y 2017 Appl. Phys. Express 10075102

[28] V.V. Afanas'ev V V, Chiappe D, Perucchini M, Houssa M, Huyghebaert C, Radu I and Stesmans A 2019 Nanotechnology 30055702

[29] Afanas'ev V V and Stesmans A 2007 J. Appl. Phys. 102 081301

[30] Afanas 'ev V V 2014 Adv. Condens. Matter. Phys. 2014 301302

[31] Afanas'ev V V 2014 Internal Photoemission Spectroscopy: Fundamentals and Recent Advances (London: Elsevier) p 385

[32] Afanas'ev V V, Schubert J, Neft A, Delie G, Shlyakhov I, Trepalin V, Houssa M and Stesmans A 2019 Microelectron. Eng. 215110992

[33] Baraskar A, Gossard A C and Rodwell M J W 2013 J. Appl. Phys. 114111605

[34] Wilk G D, Wallace R M and Anthony J M $2001 \mathrm{~J}$. Appl. Phys. 895243

[35] Das S, Chen H, Verma Penumatcha A and Appenzeller J 2013 Nano Lett.13 100

[36] Mongillo C M, Chiappe D, Arutchelvan G, Asselberghs I, Perucchini M, Manfrini M, Lin D, Huyghebaert C and Radu I 2016 Appl. Phys. Lett. 109233102

[37] Leonhardt A, Chiappe D, Asselberghs I, Hughebaert C, Radu I and De Gendt S 2017 IEEE Electron Device Lett. 38 1606

[38] Wang Y, Kim J C, Wu R J, Martinez J, Song X J, Yang J, Zhao F, Mkhoyan K A, Jeong K Y and Chhowalla M 2019 Nature $\mathbf{5 6 8} 70$

[39] Wu R J, Udyavara S, Ma R, Wang Y, Chhowalla M, Birol T, Koester S J, Neurock M and Mkhoyan K A 2019 Phys. Rev. Mater. 3111001

[40] Cui X, Lee G, Kim Y D, Arefe G, Huang P Y, Lee C, Chenet D A, Zhang X, Wang L, Ye F, Pizzocchero F, Jessen B S, Watanabe K, Taniguchi T, Muller D A, Low T, Kim P and Hone J 2015 Nature Nanotechnol. 10, 534

[41] Yoon H H, Jung S, Choi G, Kim J, Jeon Y, Kim Y S, Jeong H Y, Kim K, Kwon S-Y and Park K 2016 Nano Lett. 17 44

[42] Li Z L, Yuan K and Ye Y 2020 Nanotechnology 31 075202

[43] Alguri R and Tseng T-Y 2016 J. Electron Device Soc. 4 294

[44] Meena J S, Sze S M, Chand U and Tseng T-Y 2014 Nanoscale Res. Lett, 9526
[45] Moun M and Singh R 2018 Semicond. Sci. Technol. 33 125001

[46] McDonnell S, Addou R, Buie C, Wallace R M and Hinkle C L 2014 ACS Nano 82880

[47] Neetika, Kumar S, Sanger A, Chourasiya H K, Kumar A, Asokan K, Chandra R and Malik V K 2019 J. Alloys Compounds 797582

[48] Van Otterloo J D and Gerritsen L J 1978 J. Appl. Phys. 49723

[49] Okumura T and Tu K N 1983 J. Appl. Phys. 54922

[50] Afanas'ev V V, Kolomiiets N M, Houssa M and Stesmans A 2018 Phys. Status Solidi A 2151700865.

[51] Rangan S, Kalyanikar M, Duan J, Liu G, Bartynski R A, Andrei E Y, Feldman L and Garfunkel E 2016 J. Chem. Phys. Lett. 73434

[52] Hui F, Grustan-Guttierez E, Long S B, Liu Q, Ott A K, Ferrari A C and Lanza M 2017 Adv. Electron. Mater. 3 1600195

[53] Wang C Y, Wang C, Meng F H , Wang P F, Wang S, Liang S J and Miao F 2019 Adv. Electron. Mater. 51901107

[54] Zhu K C, Liang X H, Yan B, Villena M A, Wen C, Wang T, Chen S C, Hui F, Shi Y Y and Lanza M 2019 ACS Appl. Mater. Interfaces 1137999

[55] Paletti P, Yu R Y, Hinkle C, Fullerton-Shirley S K and Seabaugh A 2019 NPJ 2D Mater. Applications 319

[56] Yang S H, Yao Y T, Xu Y, Lin C Y, Chang Y M, Suen Y W, Sun H B, Lien C H, Li W W and Lin Y F 2019 Nanotechnology 30105201

[57] Shlyakhov I, Chai J, Yang M, Wang S J, Afanas'ev V V, Houssa M and Stesmans A 2018 APL Mater. 6026801

[58] Hong S M, Kim H-D, An H-M and Kim T G 2013 IEEE Electron Device Lett. 341181

[59] Tung R T 2001 Mater. Sci. Eng. R 351

[60] Tung R T 2014 Appl. Phys. Rev. 1011304

[61] Anderson R L 1962 Solid State Electron. 5341

[62] Afanas'ev V V, Chou H-Y, Houssa M, Stesmans A, Lamperti A, Lamagna L, Molle A, Vincent B and Brammertz G 2011 Appl. Phys. Lett. 99172101

[63] Chou H-Y, O'Connor E, Hurley P K, Afanas'ev V V, Houssa M, Stesmans A, Ye P D and Newcomb S B 2012 Appl. Phys. Lett.100 141602

[64] Liu Y, Guo J, Zhu E, Liau L, Lee S-J, Ding M, Shakir I, Gambin V, Huang Y and Duan X 2018 Nature 557696

[65] Bolshakov P, Smith C M, Khosravi A, Zhao P, Hurley P K, Hinkle C L, Wallace R M and Young C D 2019 ACS Appl. Electron. Mater. 1210

[66] Smith C M, Addou R, Hinkle C L and Wallace R M 2019 J. Phys. Chem. C 12323919

[67] Van Bremen R, Vonk K, Zandvliet H J W and Bampoulis P 2019 J. Chem. Phys. Lett. 102578

[68] McManus J B, Hennessy A, Cullin C P, Hallam T, McEvoy N and Duesberg G S 2017 Phys. Stat. Sol. B 254 1700214

[69] Salzmann B, Bernard C, Hemmi A and Grber T $2018 \mathrm{~J}$. Vac. Sci. Technol. A 36020603

[70] Pudasaini P R, Oyedele A, Zhang C, Stanford M G, Cross N, Wong A T, Hoffman A N, Xiao K, Duscher G, Mandrus D G, Ward T Z and Rack P D 2018 Nano Res. 11722 [71] Doremus R H 1971 J. Phys. Chem. 753147 
[72] Zhuravlev L T 2000 Colloids and Surfaces A 1731

[73] Wang C G and DiStefano T H 1975 CRC Crit. Rev. Solid State Sci. 5327

[74] Lang N and Kohn W 1970 Phys. Rev. B 14555

[75] Lau W M 1989 Appl. Phys. Lett. 54338

[76] Alay J L and M Hirose 1997 Appl. Phys. Lett. 811606

[77] Tanimura T, Toyoda S, Kamada H, Kumigashira H, Oshima M, Sukegawa T, Liu G L and Liu Z 2010 Appl.Phys. Lett. 96162902

[78] Prochazka P, Marecek D, Liskova Z, Cechal J and Sikola T 2017 Sci. Reports 7563

[79] Lundstrom I and DiStefano T 1976 Solid State Commun. 19871

[80] Kolomiiets N M, Afanas'ev V V, Opsomer K, Houssa M and Stesmans A 2016 Phys. Status Solidi A 213260

[81] Adamchuk V K and Afanas'ev V V 1992 Progr. Surf. Sci. 41111

[82] Powell R J 1970 J. Appl. Phys. 412424

[83] Tao J, Chai J W, Zhang Z, Pan J S and Wang S J 2014 Appl. Phys. Lett. 104232110

[84] Zhu H L, Zhou C J, Tang B S, Yang W F, Chai J W, Tai W L, Gong H, Pan J S, Zou W D, Wang S J and Chi D Z 2018 Appl. Phys. Lett. 112171604

[85] Rowe J I and Ibah H 1974 Phys. Rev. Lett.32 421

[86] Afanas'ev V, Chiappe D, Leonhardt A, Houssa M, Huyghebaert C, Radu I and Stesmans A 2017 ECS Trans. 80(1) 191

[87] Shlyakhov I, Chai J, Yang M, Wang S J, Afanas'ev V V, Houssa M and Stesmans A 2018 Phys. Staus Solidi A 216 1800616

[88] Afanas'ev V V, Chiappe D, Huyghebaert C, Radu I, De Gendt S, Houssa M and A. Stesmans A 2015 Microelectron Eng. 147294

[89] Matsukawa T, Liu Y X, Mizubayashi W, Tsukada J, Yamauchi H, Endo K, Ishikawa Y, O'uchi S, Ota H, Migita S, Morita Y and Masahara M 2013 Appl. Phys. Lett. 102162104

[90] Matsukawa T, Fukuda K, Liu Y, Tsukada J, Yamauchi H, Endo K, Ishikawa Y, O'uchi S, Migita S, Morita Y, Mizubayashi W, Ota H and Masahara M Appl. Phys. Express 8 044201

[91] Chang C C and Johnson W C 1978 IEEE Trans. Electron Dev 251368

[92] Lin H P and Hwu J G 2007 IEEE Trans. Electron Dev 54 3064

[93] Lonegran M C and Jones F E 2001 J. Chem. Phys. 115 433

[94] Bolotov L, Fukuda K, Tada T, Matsukawa T and Masahara M 2015 Jpn. J. Appl. Phys. 54 04DA03

[95] Verguts K, Coroa J, Huyghebaert C, De Gendt S and Brems S 2018 Nanoscale 105515

[96] Chiappe D, Asselberghs I, Sutar S, Iacovo S, Afanas'ev V, Stesmans A, Balaji Y, Peters L, Heyne M, Mannarino M, Vandervorst W, Sayan S, Huyghebaert C, Caymax M, Heyns M, De Gendt S, Radu I and Thean A 2015 Adv. Mater. Interfaces 31500635

[97] Heyne M H, Chiappe D, Meersschaut J, Nuytten T, Conard T, Bender H, Huyghebaert C, Radu I P, CaymaxM, de Marneffe J-F, Neyts E C and S. De Gendt 2016 J. Mater. Chemistry C 41295
[98] Chiappe D, Ludwig J, Leonhardt A, El Kazzi S, Mehta A N, Nuytten T, Celano U, Sutar S, Pourtois G, Caymax M, Paredis K, Vandervorst W, Lin D, De Gendt S, Barla K, Huyghebaert C, Asselberghs I and Radu I 2018 Nanotechnology 29425602

[99] Vos M F J, Macco B, Thissen N F W, Bol A A and Kessels W M M 2016 J. Vac. Sci. Technol. A 3401 A103

[100] Tao J, Chai J, Lu X,Wong L M, Wong T I, Pan J, Xiong Q, Chia D and Wang S J 2015 Nanoscale 72497

[101] Yan R, Zhang Q, Li W, Calizo I, Shen T, Richter C A, Hight-Walker A R, Liang X, Seabaugh A, Jena D, Xing H G, Gundlach D J and Nguyen N V 2012 Appl. Phys. Lett. 101 022105

[102] Zhang Q, Li R, Yan R, Kosel T, Xing H G, Seabaugh A C, Xu K, Kirillov O A, Gundlach D J, Richter C A and Nguyen N V 2013 Appl. Phys. Lett. 102012101

[103] Xu K, Zeng C, Zhang Q, Yan R, Ye P, Wang K, Seabaugh A C, Xing H G, Suehle J S, Richter C A, David Gundlach D J and Nguyen N V 2013 Nano Lett. 13131

[104] Li W, Zhang Q, Bijesh R, Kirillov O A, Liang Y, Levin I, Peng L-M, Richter C A, Liang X, Datta S, Gundlach D J and Nguyen N V 2014 Appl. Phys. Lett. 105213501

[105] Piskorski K, Niemiec M, Borovicz L and Przewlocki H 2017 Meas. Sci. Technol. 28055012

[106] Piskorski K, Passi V, Ruhnkopf J, Lemme M C and Przewlocki H 2018 AIP Advances 8055203

[107] Lee N J, Yoo J W, Choi Y J, Kang C J, Jeon D Y, Kim D C, Seo S and Chung H J 2009 Appl. Phys. Lett. 95222107

[108] Song S M, Park J K, Sul O J and Cho B J 2012 Nano Lett. 123887

[109] Song S M and Cho B J 2013 Carbon Lett. 14162

[110] Park J K, Song S M, Mun J H and Cho B J, 2011 Nano Lett. 115383

[111] Leonhardt A, Chiappe D, Afanas'ev V V, El Kazzi S, Shlyakhov I, Conard T, Franquet A, Hyughebaert C and de Gendt S 2019 ACS Appl. Mater. Interfaces 1142697

[112] Trepalin V, Asselberghs I, Brems S, Huyghebaert C, Radu I, Afanas'ev V, Houssa M and Stesmans A 2019 Thin Solid Films 67439

[113] Vita H, Bottcher S, Horn K, Voloshina E N, Ovcharenko R E, Kampen Th, Thissen A and Dedkov Yu S 2014 Sci. Reports 45704

[114] Ziegler D, Gava P, Guettinger J, Molitor F, Wirtz L, Lazzeri M, Saitta A M, Stemmer A, Mauri F and Stampfer 2011 Phys. Rev. B 83235434

[115] Filleter T, Emtsev K V, Seyller T and Bennewitz R 2008 Appl. Phys. Lett. 93133117

[116] Yu Y-J, Zhao Y, Ryu S, Brus L E, Kim K S and Kim P. 2009 Nano Lett. 93430

[117] Song S M, Bong J H and Cho B J 2014 Appl. Phys. Lett. 104083512

[118] An Y B, Shekhawat A, Behnam A, Pop E and Ural A 2016 Appl. Phys. Lett. 109223104

[119] Lee N J, Yoo J W, Choi Y J, Kang C J, Jeon D Y, Kim D C, Seo S and Chung H J 2009 Appl. Phys. Lett. 95222107

[120] Afanas'ev V V, Stesmans A and Andersson M O 1996 Phys. Rev. B $\mathbf{5 4} 10820$

[121] Feigl F J, Young D R, DiMaria D J, Lai S and Calise J 1981 J. Appl. Phys. 525665 
[122] Hartstein A and Young D R 1981 Appl. Phys. Lett. 38 631

[123] Solomon P M and DiMaria D J 1981 J. Appl. Phys. 52 5867

[124] Derry G N, Kern M E and Worth E H 2015 J. Vac. Sci. Technol. A 484729

[125] Peng B, Ang A K and Loh K P 2015 Nano Today 10128

[126] Zhang C, Gong C, Nie Y., Min K-A, Liang C, Oh Y J, Zhang H, Wang W, Hong S., Colombo L, Wallace R M and Cho K 2017 2D Mater. 4015026

[127] Zhang Q, Zhang S, Sperling B A and Nguyen N V 2019 J. Electron. Mater. 486446

[128] Zhang Q, Li M O, Lochocki E B, Vishwanath S, Liu X, Yan R, Lien H-H, Dobrowolska M, Furdyna J, Shen K M, Cheng G, Hight Walker A R, Gundlacj D J, Xing H J and Nguyen N V 2018 Appl. Phys. Lett. 112042108

[129] Halbritter J 1999 Appl. Phys. A 68153

[130] Freund J, Halbritter J and Horber J K H 1999 Microscopy Res. \& Techn. 44, 327

[131] Afanas'ev V V, Stesmans A, Bassler M, Pensl G, Schulz M J and Harris C I 1996 Appl. Phys. Lett. 682141

[132] Nicollian E H, Berglund C N, Schmidt P F and Andrews J M 1970 J. Appl. Phys. 413052

[133] Mahadevan T S and Garofalini S H 2008 J. Phys. Chem. C 1121507

[134] Comas-Vives A 2016 Phys. Chem. Chem. Phys. 187475

[135] Hill H M, Rigosi A F, Rim K T, Flynn G W and Heiz T F 2016 Nano Lett. 164831

[136] Shlyakhov I, Achra S, Bosman N, Asselberghs I, Huyghebaert C, Radu I, Chai J, Yang M, Wang S J, Bol A, Iakoubovskii K, Houssa M, Stesmans A and Afanas'ev V V, 2020 (in preparation).

[137] Sutar S, Agnihotri P, Comfort E, Taniguchi T, Watanabe K and Lee J U 2014 Appl. Phys. Lett. 104122104

[138] Klots A R, Newaz A K M, Wang B, Prasai D, Krzyzanowska H, Lin J, Caudel D, Ghimire N J, Yan J, Ivanov B L, Velizhanin K A, Burger A, Mandrus D G, Tolk N H, Pantelides S T and Bolotin K I 2014 Sci. Reports 46608

[139] Hill H M, Rigosi A F, Rocuelet C, Chernikov A, Berkelbach T C, Reichman D R, Hybertsen M S, Brus L E and Heiz T F 2015 Nano Lett. 152992

[140] Wang L, Wang W, Wang Q, Chi X, Kang Z, Zhou Q, Pan L, Zhang H and Wang Y 2019 RSC Advances 937195

[141] Schmidt M and Koster H 1992 Phys. Stat. Sol. B 17453

[142] Afanas'ev V V and Stesmans A 1997 Phys. Rev. Lett. 78 2437 